\title{
Representations of Quantum Affine Algebras in their $R$-Matrix Realization
}

\author{
Naihuan JING ${ }^{\dagger}$, Ming $L I U^{\ddagger}$ and Alexander MOLEV ${ }^{\S}$ \\ † Department of Mathematics, North Carolina State University, Raleigh, NC 27695, USA \\ E-mail: jing@math.ncsu.edu \\ $\ddagger$ School of Mathematics, South China University of Technology, Guangzhou, 510640, China \\ E-mail: mamliu@scut.edu.cn \\ $\S$ School of Mathematics and Statistics, University of Sydney, NSW 2006, Australia \\ E-mail: alexander.molev@sydney.edu.au
}

Received August 19, 2020, in final form December 25, 2020; Published online December 28, 2020

https://doi.org/10.3842/SIGMA.2020.145

\begin{abstract}
We use the isomorphisms between the $R$-matrix and Drinfeld presentations of the quantum affine algebras in types $B, C$ and $D$ produced in our previous work to describe finite-dimensional irreducible representations in the $R$-matrix realization. We also review the isomorphisms for the Yangians of these types and use Gauss decomposition to establish an equivalence of the descriptions of the representations in the $R$-matrix and Drinfeld presentations of the Yangians.
\end{abstract}

Key words: $R$-matrix presentation; Drinfeld polynomials; highest weight representation; Gauss decomposition

2020 Mathematics Subject Classification: 17B37

\section{Introduction}

The Yangians and quantum affine algebras associated with simple Lie algebras comprise two remarkable families of infinite-dimensional quantum groups, as introduced by V. Drinfeld [9] and M. Jimbo [17]. Both families have since found numerous connections with many areas in mathematics and physics, they possess rich and versatile representation theory.

Finite-dimensional irreducible representations of the Yangians were classified by Drinfeld in his paper [11] by relying on the pioneering work by V. Tarasov [27, 28]. The classification in [11] uses a new presentation of the Yangians and quantum affine algebras which is now often referred to as the Drinfeld presentation and which was used by V. Chari and A. Pressley to classify finitedimensional irreducible representations of quantum affine algebras [6, Chapter 12].

A different kind of presentations of these algebras known as $R$-matrix presentations goes back to the work of the Leningrad school of L. Faddeev; see, e.g., [12, 23, 25, 26]. In accordance to Drinfeld [10], such presentations can be produced from the universal $R$-matrix associated with a quantum group and they can be associated with arbitrary finite-dimensional representations. This approach was developed further in a more recent work [29].

Explicit isomorphisms between the $R$-matrix and Drinfeld presentations of the Yangians in type $A$ were given in the original work [11], while detailed proofs were produced by J. Brundan and A. Kleshchev [5]. An analogous isomorphism for the type $A$ quantum affine algebras is due to J. Ding and I. Frenkel [8].

This paper is a contribution to the Special Issue on Representation Theory and Integrable Systems in honor of Vitaly Tarasov on the 60th birthday and Alexander Varchenko on the 70th birthday. The full collection is available at https://www.emis.de/journals/SIGMA/Tarasov-Varchenko.html 
Despite their importance in representation theory and applications, such isomorphisms had remained unknown beyond type $A$ until recent work [15, 19, 20, 21], where they were produced for the remaining classical types $B, C$ and $D$.

Our goal in this paper is to give a brief review of Yangians and quantum affine algebras in types $B, C$ and $D$ and apply the isomorphisms to describe finite-dimensional irreducible representations of these algebras in their $R$-matrix realization.

In the case of Yangians, such a description was already given in [1], so that the isomorphisms connect two sides of the representation theory and explain how additional symmetries of the representation parameters arise from the Gauss decomposition of the generator matrices. However, for the $R$-matrix realization of the quantum affine algebras the isomorphisms are essential to get a parametrization of their finite-dimensional irreducible representations.

A key step in our arguments relies on a consistency property for the triangular decomposition of the algebra and the Gauss decomposition of the generator matrices. The property ensures that the upper triangular Gaussian generators can be expressed in terms of the 'simple root generators' implying that the annihilation properties of the highest vectors agree in both presentations of the algebras. Its Yangian version is well-known in type $A$ (see, e.g., [24, Section 3.1]), while proofs in the remaining classical types follow from [19, Lemma 5.15]. This property has also been known for the quantum affine algebras of type $A$ [22], so that the isomorphism of [8] connects the descriptions of the representations given in [6, Section 12.2] and [14]. Similar to the approach of [22], we establish the consistency property in Section 3.2.4 in types $B, C$ and $D$ by deriving it from the defining relations. An alternative way can rely on explicit formulas for the universal $R$-matrices which, however, leads to more involved calculations.

In Appendix A we give a modified version of the Yangian isomorphisms produced in [19], which is based on the opposite Gauss decomposition of the generator matrix. This version can be applied to make an alternative connection between the parameters of representations in the two realizations of the Yangians.

\section{Representations of Yangians of types $B, C$ and $D$}

\subsection{Classification results}

We will denote by $\mathfrak{g}$ one of the simple Lie algebras of type $B_{n},{ }_{n}$ or $D_{n}$. That is, $\mathfrak{g}=\mathfrak{o}_{N}$ is either the orthogonal Lie algebra with $N=2 n+1$ and $N=2 n$, or $\mathfrak{g}=\mathfrak{s p}_{N}$ is the symplectic Lie algebra with $N=2 n$. Choose simple roots in the form

$$
\alpha_{i}=\epsilon_{i}-\epsilon_{i+1} \quad \text { for } \quad i=1, \ldots, n-1,
$$

and

$$
\alpha_{n}= \begin{cases}\epsilon_{n} & \text { if } \mathfrak{g}=\mathfrak{o}_{2 n+1} \\ 2 \epsilon_{n} & \text { if } \mathfrak{g}=\mathfrak{s p}_{2 n}, \\ \epsilon_{n-1}+\epsilon_{n} & \text { if } \mathfrak{g}=\mathfrak{o}_{2 n},\end{cases}
$$

where $\epsilon_{1}, \ldots, \epsilon_{n}$ is an orthonormal basis of a Euclidian space with the inner product $(\cdot, \cdot)$. The Cartan matrix $\left[A_{i j}\right]$ for $\mathfrak{g}$ is given by

$$
A_{i j}=\frac{2\left(\alpha_{i}, \alpha_{j}\right)}{\left(\alpha_{i}, \alpha_{i}\right)}
$$

We will use the notation

$$
r_{i}=\frac{1}{2}\left(\alpha_{i}, \alpha_{i}\right), \quad i=1, \ldots, n .
$$


As defined in [11], the Drinfeld Yangian $\mathrm{Y}^{D}(\mathfrak{g})$ is generated by elements $\kappa_{i r}, \xi_{i r}^{+}$and $\xi_{i r}^{-}$with $i=1, \ldots, n$ and $r=0,1, \ldots$ subject to the defining relations

$$
\begin{aligned}
& {\left[\kappa_{i r}, \kappa_{j s}\right]=0,} \\
& {\left[\xi_{i r}^{+}, \xi_{j s}^{-}\right]=\delta_{i j} \kappa_{i r+s},} \\
& {\left[\kappa_{i 0}, \xi_{j s}^{ \pm}\right]= \pm\left(\alpha_{i}, \alpha_{j}\right) \xi_{j s}^{ \pm},} \\
& {\left[\kappa_{i r+1}, \xi_{j s}^{ \pm}\right]-\left[\kappa_{i r}, \xi_{j s+1}^{ \pm}\right]= \pm \frac{\left(\alpha_{i}, \alpha_{j}\right)}{2}\left(\kappa_{i r} \xi_{j s}^{ \pm}+\xi_{j s}^{ \pm} \kappa_{i r}\right),} \\
& {\left[\xi_{i r+1}^{ \pm}, \xi_{j s}^{ \pm}\right]-\left[\xi_{i r}^{ \pm}, \xi_{j s+1}^{ \pm}\right]= \pm \frac{\left(\alpha_{i}, \alpha_{j}\right)}{2}\left(\xi_{i r}^{ \pm} \xi_{j s}^{ \pm}+\xi_{j s}^{ \pm} \xi_{i r}^{ \pm}\right),} \\
& \sum_{p \in \mathfrak{S}_{m}}\left[\xi_{i r_{p(1)}}^{ \pm},\left[\xi_{i r_{p(2)}}^{ \pm}, \ldots\left[\xi_{i r_{p(m)}}^{ \pm}, \xi_{j s}^{ \pm}\right] \ldots\right]\right]=0,
\end{aligned}
$$

where the last relation holds for all $i \neq j$, and we denoted $m=1-a_{i j}$.

By the results of [11] (they apply to any simple Lie algebra $\mathfrak{g}$ ), every finite-dimensional irreducible representation of the algebra $\mathrm{Y}^{D}(\mathfrak{g})$ is generated by a nonzero vector $\zeta$ (called the highest vector) which is annihilated by all $\xi_{i r}^{+}$and is a simultaneous eigenvector for all $\kappa_{i r}$ so that

$$
\kappa_{i r} \zeta=d_{i r} \zeta, \quad d_{i r} \in \mathbb{C}
$$

Furthermore, there exist unique monic polynomials $P_{1}(u), \ldots, P_{n}(u)$ in $u$ such that

$$
1+\sum_{r=0}^{\infty} d_{i r} u^{-r-1}=\frac{P_{i}\left(u+r_{i}\right)}{P_{i}(u)}, \quad i=1, \ldots, n .
$$

Equivalently, every finite-dimensional irreducible representation of the algebra $\mathrm{Y}^{D}(\mathfrak{g})$ is generated by a nonzero vector $\zeta^{\prime}$ (the highest vector with respect to the opposite triangular decomposition) which is annihilated by all $\xi_{i r}^{-}$and is a simultaneous eigenvector for all $\kappa_{i r}$ so that

$$
\kappa_{i r} \zeta^{\prime}=d_{i r}^{\prime} \zeta^{\prime}, \quad d_{i r}^{\prime} \in \mathbb{C}
$$

Furthermore, there exist unique monic polynomials $Q_{1}(u), \ldots, Q_{n}(u)$ in $u$ such that

$$
1+\sum_{r=0}^{\infty} d_{i r}^{\prime} u^{-r-1}=\frac{Q_{i}(u)}{Q_{i}\left(u+r_{i}\right)}, \quad i=1, \ldots, n .
$$

All possible $n$-tuples of monic polynomials $\left(P_{1}(u), \ldots, P_{n}(u)\right)$ and $\left(Q_{1}(u), \ldots, Q_{n}(u)\right)$ arise in this way. The equivalence of the two parametrizations is seen by using the automorphism of the algebra $\mathrm{Y}^{D}(\mathfrak{g})$ defined by

$$
\xi_{i r}^{+} \mapsto(-1)^{r+1} \xi_{i r}^{-}, \quad \xi_{i r}^{-} \mapsto(-1)^{r+1} \xi_{i r}^{+}, \quad \kappa_{i r} \mapsto(-1)^{r+1} \kappa_{i r} .
$$

\subsection{Gaussian generators and isomorphisms}

Introduce the following elements of the endomorphism algebra End $\mathbb{C}^{N} \otimes$ End $\mathbb{C}^{N}$ :

$$
P=\sum_{i, j=1}^{N} e_{i j} \otimes e_{j i} \quad \text { and } \quad Q=\sum_{i, j=1}^{N} \varepsilon_{i} \varepsilon_{j} e_{i j} \otimes e_{i^{\prime} j^{\prime}}
$$


where $e_{i j} \in$ End $\mathbb{C}^{N}$ are the matrix units, and we use the notation $i^{\prime}=N+1-i$ and set $\varepsilon_{i} \equiv 1$ in the orthogonal case, and

$$
\varepsilon_{i}=\left\{\begin{aligned}
1 & \text { for } i=1, \ldots, n \\
-1 & \text { for } i=n+1, \ldots, 2 n
\end{aligned}\right.
$$

in the symplectic case. Set

$$
\kappa= \begin{cases}N / 2-1 & \text { in the orthogonal case } \\ N / 2+1 & \text { in the symplectic case }\end{cases}
$$

Following [30], consider the $R$-matrix $R(u)$

$$
R(u)=1-\frac{P}{u}+\frac{Q}{u-\kappa} .
$$

The extended Yangian $\mathrm{X}(\mathfrak{g})$ is defined as a unital associative algebra with generators $t_{i j}^{(r)}$, where $1 \leqslant i, j \leqslant N$ and $r=1,2, \ldots$, satisfying certain quadratic relations. Introduce the formal series

$$
t_{i j}(u)=\delta_{i j}+\sum_{r=1}^{\infty} t_{i j}^{(r)} u^{-r} \in \mathrm{X}(\mathfrak{g})\left[\left[u^{-1}\right]\right]
$$

and set

$$
T(u)=\sum_{i, j=1}^{N} e_{i j} \otimes t_{i j}(u) \in \operatorname{End} \mathbb{C}^{N} \otimes \mathrm{X}(\mathfrak{g})\left[\left[u^{-1}\right]\right] .
$$

Consider the tensor product algebra End $\mathbb{C}^{N} \otimes \operatorname{End} \mathbb{C}^{N} \otimes \mathrm{X}(\mathfrak{g})$ and introduce the series with coefficients in this algebra by

$$
T_{1}(u)=\sum_{i, j=1}^{N} e_{i j} \otimes 1 \otimes t_{i j}(u) \quad \text { and } \quad T_{2}(u)=\sum_{i, j=1}^{N} 1 \otimes e_{i j} \otimes t_{i j}(u) .
$$

The defining relations for the algebra $\mathrm{X}(\mathfrak{g})$ are then written in the form

$$
R(u-v) T_{1}(u) T_{2}(v)=T_{2}(v) T_{1}(u) R(u-v) .
$$

The Yangian $\mathrm{Y}(\mathfrak{g})$ is defined as the subalgebra of $\mathrm{X}(\mathfrak{g})$ which consists of the elements stable under the automorphisms

$$
\mu_{f}: T(u) \mapsto f(u) T(u)
$$

for all series $f(u)=1+f_{1} u^{-1}+f_{2} u^{-2}+\cdots$ with $f_{i} \in \mathbb{C}$. Equivalently, $\mathrm{Y}(\mathfrak{g})$ is isomorphic to the quotient of the algebra $\mathrm{X}(\mathfrak{g})$ by the relation

$$
T^{\mathrm{t}}(u+\kappa) T(u)=1,
$$

where t denotes the matrix transposition with $e_{i j}^{\mathrm{t}}=\varepsilon_{i} \varepsilon_{j} e_{j^{\prime}, i^{\prime}}$. 


\subsubsection{Isomorphisms}

Apply the Gauss decomposition to the matrix $T(u)$,

$$
T(u)=F(u) H(u) E(u),
$$

where $F(u), H(u)$ and $E(u)$ are uniquely determined matrices of the form

$$
F(u)=\left[\begin{array}{cccc}
1 & 0 & \ldots & 0 \\
f_{21}(u) & 1 & \ldots & 0 \\
\vdots & \vdots & \ddots & \vdots \\
f_{N 1}(u) & f_{N 2}(u) & \ldots & 1
\end{array}\right], \quad E(u)=\left[\begin{array}{cccc}
1 & e_{12}(u) & \ldots & e_{1 N}(u) \\
0 & 1 & \ldots & e_{2 N}(u) \\
\vdots & \vdots & \ddots & \vdots \\
0 & 0 & \ldots & 1
\end{array}\right]
$$

and $H(u)=\operatorname{diag}\left[h_{1}(u), \ldots, h_{N}(u)\right]$. Define the series with coefficients in $\mathrm{Y}(\mathfrak{g})$ by

$$
\kappa_{i}(u)=h_{i}(u-(i-1) / 2)^{-1} h_{i+1}(u-(i-1) / 2)
$$

for $i=1, \ldots, n-1$, and

$$
\kappa_{n}(u)= \begin{cases}h_{n}(u-(n-1) / 2)^{-1} h_{n+1}(u-(n-1) / 2) & \text { for } \mathfrak{o}_{2 n+1}, \\ h_{n}(u-n / 2)^{-1} h_{n+1}(u-n / 2) & \text { for } \mathfrak{s p}_{2 n}, \\ h_{n-1}(u-(n-2) / 2)^{-1} h_{n+1}(u-(n-2) / 2) & \text { for } \mathfrak{o}_{2 n} .\end{cases}
$$

Furthermore, set

$$
\xi_{i}^{+}(u)=f_{i+1 i}(u-(i-1) / 2), \quad \xi_{i}^{-}(u)=e_{i i+1}(u-(i-1) / 2)
$$

for $i=1, \ldots, n-1$,

$$
\xi_{n}^{+}(u)= \begin{cases}f_{n+1 n}(u-(n-1) / 2) & \text { for } \mathfrak{o}_{2 n+1}, \\ f_{n+1 n}(u-n / 2) & \text { for } \mathfrak{s p}_{2 n}, \\ f_{n+1 n-1}(u-(n-2) / 2) & \text { for } \mathfrak{o}_{2 n}\end{cases}
$$

and

$$
\xi_{n}^{-}(u)= \begin{cases}e_{n n+1}(u-(n-1) / 2) & \text { for } \mathfrak{o}_{2 n+1}, \\ \frac{1}{2} e_{n n+1}(u-n / 2) & \text { for } \mathfrak{s p}_{2 n} \\ e_{n-1 n+1}(u-(n-2) / 2) & \text { for } \mathfrak{o}_{2 n}\end{cases}
$$

Introduce elements of $\mathrm{Y}(\mathfrak{g})$ by the respective expansions into power series in $u^{-1}$,

$$
\kappa_{i}(u)=1+\sum_{r=0}^{\infty} \kappa_{i r} u^{-r-1} \quad \text { and } \quad \xi_{i}^{ \pm}(u)=\sum_{r=0}^{\infty} \xi_{i r}^{ \pm} u^{-r-1}
$$

for $i=1, \ldots, n$. According to [19, Main Theorem], the mapping which sends the generators $\kappa_{i r}$ and $\xi_{i r}^{ \pm}$of $\mathrm{Y}^{D}(\mathfrak{g})$ to the elements of $\mathrm{Y}(\mathfrak{g})$ with the same names defines an isomorphism $\mathrm{Y}^{D}(\mathfrak{g}) \cong \mathrm{Y}(\mathfrak{g})$. 


\subsubsection{Central elements of the extended Yangian}

A presentation of the extended Yangian $\mathrm{X}(\mathfrak{g})$ in terms of the Gaussian generators is given in [19, Theorem 5.14]. ${ }^{1}$ By [19, Theorem 5.8], all coefficients of the series

$$
z(u)= \begin{cases}\prod_{i=1}^{n} h_{i}(u+\kappa-i)^{-1} \prod_{i=1}^{n} h_{i}(u+\kappa-i+1) \cdot h_{n+1}(u) h_{n+1}(u-1 / 2) & \text { if } N=2 n+1, \\ \prod_{i=1}^{n-1} h_{i}(u+\kappa-i)^{-1} \prod_{i=1}^{n} h_{i}(u+\kappa-i+1) \cdot h_{n+1}(u) & \text { if } N=2 n,\end{cases}
$$

belong to the center $\mathcal{C}$ of the extended Yangian $\mathrm{X}(\mathfrak{g})$. Moreover, these coefficients generate the center and we have the tensor product decomposition

$$
\mathrm{X}(\mathfrak{g}) \cong \mathrm{Y}(\mathfrak{g}) \otimes \mathcal{C}
$$

The following identity holds in $\mathrm{X}(\mathfrak{g})$ :

$$
T^{\mathrm{t}}(u+\kappa) T(u)=z(u)
$$

so that the Yangian $\mathrm{Y}(\mathfrak{g})$ is isomorphic to the quotient of $\mathrm{X}(\mathfrak{g})$ by the relation $z(u)=1$. We will record the relations which follow from the arguments in [19, Section 5.3].

Lemma 2.1. In the algebra $\mathrm{X}(\mathfrak{g})$ we have

$$
\begin{aligned}
& h_{1}(u+\kappa) h_{1^{\prime}}(u)=z(u), \\
& h_{i}(u+\kappa-i) h_{i^{\prime}}(u)=h_{i+1}(u+\kappa-i) h_{(i+1)^{\prime}}(u),
\end{aligned}
$$

where $i=1, \ldots, n$ for $N=2 n+1$, and $i=1, \ldots, n-1$ for $N=2 n$.

\subsection{Highest weight representations}

Definition 2.2. A representation $V$ of the algebra $\mathrm{Y}(\mathfrak{g})($ or $\mathrm{X}(\mathfrak{g}))$ is called a highest weight representation if there exists a nonzero vector $\zeta \in V$ such that $V$ is generated by $\zeta$ and the following relations hold:

$$
\begin{array}{ll}
t_{i j}(u) \zeta=0 & \text { for } \quad 1 \leqslant i<j \leqslant N, \quad \text { and } \\
t_{i i}(u) \zeta=\lambda_{i}(u) \zeta & \text { for } \quad i=1, \ldots, N,
\end{array}
$$

for some formal series $\lambda_{i}(u) \in 1+u^{-1} \mathbb{C}\left[\left[u^{-1}\right]\right]$. The vector $\zeta$ is called the highest vector of the representation $V$.

The following classification theorem for finite-dimensional irreducible representations of the Yangians in types $B, C$ and $D$ was proved in [1] in terms of their $R$-matrix presentation. We will use the isomorphisms of [19] which we recalled in Section 2.2.1, to make an explicit connection between this theorem and the results of [11]. Note that such a connection was already established in [15], where isomorphisms between three presentations of the orthogonal and symplectic Yangians were constructed. However, those results did not use the Gaussian presentation which we will rely on in our arguments.

\footnotetext{
${ }^{1}$ Note that the formulas in [19, equations (5.4) and (5.47)] should be corrected by swapping the order of the factors on their right hand sides.
} 


\section{Theorem 2.3.}

1. Any finite-dimensional irreducible representation of the algebra $\mathrm{Y}(\mathfrak{g})$ is a highest weight representation. Its parameters satisfy the relations

$$
\frac{\lambda_{i}(u)}{\lambda_{i+1}(u)}=\frac{P_{i}(u+1)}{P_{i}(u)}, \quad i=1, \ldots, n-1,
$$

and

$$
\begin{array}{rlrl}
\frac{\lambda_{n}(u)}{\lambda_{n+1}(u)}=\frac{P_{n}(u+1 / 2)}{P_{n}(u)} & & \text { for type } B_{n}, \\
\frac{\lambda_{n}(u)}{\lambda_{n+1}(u)}=\frac{P_{n}(u+2)}{P_{n}(u)} & & \text { for type } C_{n}, \\
\frac{\lambda_{n-1}(u)}{\lambda_{n+1}(u)}=\frac{P_{n}(u+1)}{P_{n}(u)} & \text { for type } D_{n},
\end{array}
$$

for some monic polynomials $P_{i}(u)$ in $u$.

2. Every $n$-tuple $\left(P_{1}(u), \ldots, P_{n}(u)\right)$ of monic polynomials in $u$ arises in this way.

3. The series $\lambda_{i}(u)$ satisfy the relations

$$
\lambda_{i}(u+\kappa-i) \lambda_{i^{\prime}}(u)=\lambda_{i+1}(u+\kappa-i) \lambda_{(i+1)^{\prime}}(u),
$$

where $i=0,1, \ldots, n$ for $N=2 n+1$, and $i=0,1, \ldots, n-1$ for $N=2 n$, and we set $\lambda_{0}(u)=\lambda_{0^{\prime}}(u):=1$.

Proof. Using the isomorphism $\mathrm{Y}^{D}(\mathfrak{g}) \cong \mathrm{Y}(\mathfrak{g})$ and the classification results recalled in Section 2.1, we find that any finite-dimensional irreducible representation $V$ of the algebra $\mathrm{Y}(\mathfrak{g})$ in types $B_{n}$ and $C_{n}$ is generated by a vector $\zeta^{\prime}$ such that

$$
\begin{array}{ll}
e_{i, i+1}(u) \zeta^{\prime}=0 & \text { for } \quad i=1, \ldots, n, \\
h_{i}(u) \zeta^{\prime}=\lambda_{i}(u) \zeta^{\prime} & \text { for } \quad i=1, \ldots, n+1,
\end{array}
$$

for some formal series $\lambda_{i}(u) \in 1+u^{-1} \mathbb{C}\left[\left[u^{-1}\right]\right]$. For type $D_{n}$, the same conditions hold, except that relation (2.9) with $i=n$ should be replaced with $e_{n-1, n+1}(u) \zeta^{\prime}=0$. Indeed, for all types, relation (2.9) is clear from the definition of the highest vector $\zeta^{\prime}$, while (2.10) follows from the condition that $\zeta^{\prime}$ is an eigenvector for all series $\kappa_{i}(u)$ with $i=1, \ldots, n$ and $z(u) \zeta^{\prime}=\zeta^{\prime}$. Now, Lemma 2.1 and [19, Lemma 5.15] imply that

$$
\begin{array}{ll}
e_{i j}(u) \zeta^{\prime}=0 & \text { for } \quad i<j \\
h_{i}(u) \zeta^{\prime}=\lambda_{i}(u) \zeta^{\prime} & \text { for } \quad i=1, \ldots, N,
\end{array}
$$

for certain formal series $\lambda_{i}(u) \in 1+u^{-1} \mathbb{C}\left[\left[u^{-1}\right]\right]$ satisfying identities (2.8). Finally, note that the values of the parameters (2.3) are found by $r_{i}=1$ for $i=1, \ldots, n-1$, while $r_{n}=1 / 2$ for type $B_{n}, r_{n}=2$ for type $C_{n}$, and $r_{n}=1$ for type $D_{n}$, so that conditions in Part 1 of the theorem follow from (2.4).

Corollary 2.4. All statements of Theorem 2.3 hold in the same form for the extended Yangian $\mathrm{X}(\mathfrak{g})$, except that the value $i=0$ is excluded for the conditions (2.8).

Proof. The proof of the theorem obviously extends to the algebra $\mathrm{X}(\mathfrak{g})$. Relation (2.8) with $i=0$ is now replaced by the property that the series $z(u)$ acts in the highest weight representation as multiplication by $\lambda_{1}(u+\kappa) \lambda_{1^{\prime}}(u)$.

Remark 2.5. It is clear that the arguments used in the proof of the theorem can be reversed, so that the classification theorem for the Yangian representations in types $B, C$ and $D$ proved in [1] implies the corresponding results of [11]. 


\section{Representations of quantum affine algebras}

\subsection{Classification results}

We will suppose that $q$ is a nonzero complex number which is not a root of unity and set $q_{i}=q^{r_{i}}$ for $i=1, \ldots, n$, with $r_{i}$ defined in (2.3). The Cartan matrix of the simple Lie algebra $\mathfrak{g}$ of type $B_{n}, C_{n}$ or $D_{n}$ is given by (2.2). We will use the standard notation

$$
[k]_{q}=\frac{q^{k}-q^{-k}}{q-q^{-1}}
$$

for a nonnegative integer $k$, together with

$$
[k]_{q} !=\prod_{s=1}^{k}[s]_{q} \quad \text { and } \quad\left[\begin{array}{l}
k \\
r
\end{array}\right]_{q}=\frac{[k]_{q} !}{[r]_{q} ![k-r]_{q} !} .
$$

The quantum affine algebra $\mathrm{U}_{q}(\widehat{\mathfrak{g}})$ (with the trivial central charge) in its Drinfeld presentation is the associative algebra with generators $x_{i, m}^{ \pm}, a_{i, l}$ and $k_{i}^{ \pm 1}$ for $i=1, \ldots, n$ and $m, l \in \mathbb{Z}$ with $l \neq 0$, subject to the following defining relations:

$$
\begin{aligned}
& k_{i} k_{j}=k_{j} k_{i}, \quad k_{i} a_{j, l}=a_{j, l} k_{i}, \quad a_{i, m} a_{j, l}=a_{j, l} a_{i, m}, \\
& k_{i} x_{j, m}^{ \pm} k_{i}^{-1}=q_{i}^{ \pm A_{i j}} x_{j, m}^{ \pm}, \quad\left[a_{i, m}, x_{j, l}^{ \pm}\right]= \pm \frac{\left[m A_{i j}\right]_{q_{i}}}{m} x_{j, m+l}^{ \pm}, \\
& x_{i, m+1}^{ \pm} x_{j, l}^{ \pm}-q_{i}^{ \pm A_{i j}} x_{j, l}^{ \pm} x_{i, m+1}^{ \pm}=q_{i}^{ \pm A_{i j}} x_{i, m}^{ \pm} x_{j, l+1}^{ \pm}-x_{j, l+1}^{ \pm} x_{i, m}^{ \pm}, \\
& {\left[x_{i, m}^{+}, x_{j, l}^{-}\right]=\delta_{i j} \frac{\psi_{i, m+l}-\varphi_{i, m+l}}{q_{i}-q_{i}^{-1}},} \\
& \sum_{\pi \in \mathfrak{S}_{r}} \sum_{l=0}^{r}(-1)^{l}\left[\begin{array}{c}
r \\
l
\end{array}\right]_{q_{i}} x_{i, s_{\pi(1)}}^{ \pm} \cdots x_{i, s_{\pi(l)}}^{ \pm} x_{j, m}^{ \pm} x_{i, s_{\pi(l+1)}}^{ \pm} \cdots x_{i, s_{\pi(r)}}^{ \pm}=0, \quad i \neq j,
\end{aligned}
$$

where in the last relation we set $r=1-A_{i j}$. The elements $\psi_{i, m}$ and $\varphi_{i,-m}$ with $m \in \mathbb{Z}_{+}$are defined by

$$
\begin{aligned}
& \psi_{i}(u):=\sum_{m=0}^{\infty} \psi_{i, m} u^{-m}=k_{i} \exp \left(\left(q_{i}-q_{i}^{-1}\right) \sum_{s=1}^{\infty} a_{i, s} u^{-s}\right), \\
& \varphi_{i}(u):=\sum_{m=0}^{\infty} \varphi_{i,-m} u^{m}=k_{i}^{-1} \exp \left(-\left(q_{i}-q_{i}^{-1}\right) \sum_{s=1}^{\infty} a_{i,-s} u^{s}\right),
\end{aligned}
$$

whereas $\psi_{i, m}=\varphi_{i,-m}=0$ for $m<0$.

Remark 3.1. For the Lie algebras $\mathfrak{g}$ of types $C_{n}$ and $D_{n}$, it will be convenient to work with an extended quantum algebra obtained by adjoining the square roots $k_{n}^{ \pm 1 / 2}$ and $\left(k_{n-1} k_{n}\right)^{ \pm 1 / 2}$, respectively. Accordingly, we need to add the defining relations

$$
k_{n}^{1 / 2} x_{j, m}^{ \pm} k_{n}^{-1 / 2}=q^{ \pm A_{n j}} x_{j, m}^{ \pm}
$$

for type $C_{n}$, and

$$
\left(k_{n-1} k_{n}\right)^{1 / 2} x_{j, m}^{ \pm}\left(k_{n-1} k_{n}\right)^{-1 / 2}=q^{ \pm\left(A_{n-1, j}+A_{n j}\right) / 2} x_{j, m}^{ \pm}
$$

for type $D_{n}$, while the new elements commute with all the remaining generators. 
As explained in [6, Section 12.2], the algebra $\mathrm{U}_{q}(\widehat{\mathfrak{g}})$ admits a family of sign automorphisms such that the composition of any finite-dimensional irreducible representation with a suitable automorphism of this kind is isomorphic to a type $\mathbf{1}$ representation. Such a representation is generated by a vector $\zeta$ which is annihilated by all $x_{i, m}^{+}$and is a simultaneous eigenvector for all $k_{i}$ and $a_{i, l}$. Furthermore, if the series $\Phi_{i}(u) \in \mathbb{C}[[u]]$ and $\Psi_{i}(u) \in \mathbb{C}\left[\left[u^{-1}\right]\right]$ are defined by

$$
\varphi_{i}(u) \zeta=\Phi_{i}(u) \zeta \quad \text { and } \quad \psi_{i}(u) \zeta=\Psi_{i}(u) \zeta
$$

then there exist unique polynomials $P_{1}(u), \ldots, P_{n}(u)$ in $u$ all with constant term 1 such that

$$
\Phi_{i}(u)=q_{i}^{-\operatorname{deg} P_{i}} \frac{P_{i}\left(u q_{i}^{2}\right)}{P_{i}(u)}=\Psi_{i}(u), \quad i=1, \ldots, n,
$$

where the equalities are understood for the expansions of the rational functions in $u$ as series in $u$ and $u^{-1}$, respectively. ${ }^{2}$ Every $n$-tuple of polynomials $\left(P_{1}(u), \ldots, P_{n}(u)\right)$ in $u$, where each $P_{i}(u)$ has constant term 1 , arises in this way.

The above classification of finite-dimensional irreducible representations of $\mathrm{U}_{q}(\widehat{\mathfrak{g}})$ is valid for any simple Lie algebra $\mathfrak{g}$. Note also that the corresponding results of [6, Section 12.2] apply to centrally extended algebras $\mathrm{U}_{q}(\widehat{\mathfrak{g}})$, which show that the central element acts trivially in any finite-dimensional representation. For this reason we only consider the quotients of the quantum affine algebras by the relation specifying the value of the central element as equal to 1 .

\subsection{Gaussian generators and isomorphisms}

To define $R$-matrix realizations of the quantum affine algebras, introduce elements of the endomorphism algebra End $\mathbb{C}^{N} \otimes$ End $\mathbb{C}^{N}$ by

$$
P=\sum_{i, j=1}^{N} e_{i j} \otimes e_{j i} \quad \text { and } \quad Q=\sum_{i, j=1}^{N} q^{\bar{\imath}-\bar{\jmath}} \varepsilon_{i} \varepsilon_{j} e_{i^{\prime} j^{\prime}} \otimes e_{i j},
$$

where $i^{\prime}=N+1-i$, as before, and

$$
(\overline{1}, \overline{2}, \ldots, \bar{N})= \begin{cases}\left(n-\frac{1}{2}, \ldots, \frac{3}{2}, \frac{1}{2}, 0,-\frac{1}{2},-\frac{3}{2}, \ldots,-n+\frac{1}{2}\right) & \text { for } \mathfrak{g}=\mathfrak{o}_{2 n+1} \\ (n, \ldots, 2,1,-1,-2, \ldots,-n) & \text { for } \mathfrak{g}=\mathfrak{s p}_{2 n} \\ (n-1, \ldots, 1,0,0,-1, \ldots,-n+1) & \text { for } \mathfrak{g}=\mathfrak{o}_{2 n}\end{cases}
$$

Furthermore, introduce the $R$-matrix by

$$
\begin{aligned}
R= & q \sum_{i=1, i \neq i^{\prime}}^{N} e_{i i} \otimes e_{i i}+e_{n+1, n+1} \otimes e_{n+1, n+1}+\sum_{i \neq j, j^{\prime}} e_{i i} \otimes e_{j j}+q^{-1} \sum_{i \neq i^{\prime}} e_{i i} \otimes e_{i^{\prime} i^{\prime}} \\
& +\left(q-q^{-1}\right) \sum_{i<j} e_{i j} \otimes e_{j i}-\left(q-q^{-1}\right) \sum_{i>j} q^{\bar{\imath}-\bar{\jmath}} \varepsilon_{i} \varepsilon_{j} e_{i^{\prime} j^{\prime}} \otimes e_{i j},
\end{aligned}
$$

where the second term $e_{n+1, n+1} \otimes e_{n+1, n+1}$ should be omitted if $N=2 n$. This formula goes back to $[2,3,18,26]$, which appeared along with the spectral parameter-dependent $R$-matrix

$$
R(u)=(u-1) R+\left(q-q^{-1}\right)\left(P-\frac{u \xi-\xi}{u-\xi} Q\right)
$$

\footnotetext{
${ }^{2}$ The roles of $u$ and $u^{-1}$ are swapped in our notation as compared to [6, Section 12.2].
} 
where

$$
\xi=q^{-2 \kappa}= \begin{cases}q^{-N+2} & \text { if } \mathfrak{g}=\mathfrak{o}_{N} \\ q^{-N-2} & \text { if } \mathfrak{g}=\mathfrak{s p}_{2 n}\end{cases}
$$

The corresponding two-parameter $R$-matrix $R(u, v)=v R(u / v)$ can be written as

$$
\begin{aligned}
R(u, v)= & \sum_{i, j=1}^{N}\left(u q^{\delta_{i j}}-v q^{-\delta_{i j}}\right) e_{i i} \otimes e_{j j}+\left(q-q^{-1}\right) \sum_{i, j=1}^{N}\left(u \delta_{i<j}+v \delta_{i>j}\right) e_{i j} \otimes e_{j i} \\
& -\frac{u-v}{u-v \xi} \sum_{i, j=1}^{N} d_{i j}(u, v) e_{i^{\prime} j^{\prime}} \otimes e_{i j},
\end{aligned}
$$

where $\delta_{i<j}$ or $\delta_{i>j}$ equals 1 if the subscript inequality holds, and 0 otherwise, and

$$
d_{i j}(u, v)= \begin{cases}\left(q-q^{-1}\right) \xi v q^{\bar{\imath}-\bar{\jmath}} \varepsilon_{i} \varepsilon_{j} & \text { for } i<j, \\ \left(q-q^{-1}\right) u q^{\bar{\imath}-\bar{\jmath}} \varepsilon_{i} \varepsilon_{j} & \text { for } i>j, \\ \left(1-q^{-1}\right)(u+v q \xi) & \text { for } i=j, i \neq i^{\prime}, \\ \left(1-q^{-1}\right)(u q+v \xi) & \text { for } i=j=i^{\prime} .\end{cases}
$$

Observe that by setting $d_{i j}(u, v) \equiv 0$ we recover the trigonometric $R$-matrix for type $A$. Note also that if $N=2 n$, then the equality $i=i^{\prime}$ is impossible, so that the last case in the definition of $d_{i j}(u, v)$ does not occur.

The quantum affine algebra $\mathrm{U}_{q}^{R}(\widehat{\mathfrak{g}})$ (with the trivial central charge) is generated by the elements $l_{i j}^{ \pm}[\mp m]$ with $1 \leqslant i, j \leqslant N$ and $m \in \mathbb{Z}_{+}$subject to the following defining relations. We have $^{3}$

$$
l_{i j}^{+}[0]=l_{j i}^{-}[0]=0 \quad \text { for } \quad i<j \quad \text { and } \quad l_{i i}^{+}[0] l_{i i}^{-}[0]=l_{i i}^{-}[0] l_{i i}^{+}[0]=1,
$$

while the remaining relations will be written in terms of the formal power series

$$
l_{i j}^{ \pm}(u)=\sum_{m=0}^{\infty} l_{i j}^{ \pm}[\mp m] u^{ \pm m}
$$

combined into the respective matrices

$$
L^{ \pm}(u)=\sum_{i, j=1}^{N} e_{i j} \otimes l_{i j}^{ \pm}(u) \in \operatorname{End} \mathbb{C}^{N} \otimes \mathrm{U}_{q}^{R}(\widehat{\mathfrak{g}})\left[\left[u, u^{-1}\right]\right] .
$$

Consider the tensor product algebra End $\mathbb{C}^{N} \otimes$ End $\mathbb{C}^{N} \otimes \mathrm{U}_{q}^{R}(\widehat{\mathfrak{g}})$ and introduce the series with coefficients in this algebra by

$$
L_{1}^{ \pm}(u)=\sum_{i, j=1}^{N} e_{i j} \otimes 1 \otimes l_{i j}^{ \pm}(u) \quad \text { and } \quad L_{2}^{ \pm}(u)=\sum_{i, j=1}^{N} 1 \otimes e_{i j} \otimes l_{i j}^{ \pm}(u) .
$$

The defining relations then take the form

$$
\begin{aligned}
& R(u, v) L_{1}^{ \pm}(u) L_{2}^{ \pm}(v)=L_{2}^{ \pm}(v) L_{1}^{ \pm}(u) R(u, v), \\
& R(u, v) L_{1}^{+}(u) L_{2}^{-}(v)=L_{2}^{-}(v) L_{1}^{+}(u) R(u, v),
\end{aligned}
$$

together with the relations

$$
L^{ \pm}(u) D L^{ \pm}(u \xi)^{\mathrm{t}} D^{-1}=1,
$$

where $D$ is the diagonal matrix

$$
D=\operatorname{diag}\left[q^{\overline{1}}, \ldots, q^{\bar{N}}\right] \text {. }
$$

\footnotetext{
${ }^{3}$ The condition $i<j$ was erroneously replaced by the opposite inequality in [20, 21].
} 


\subsubsection{Isomorphisms}

By applying the Gauss decomposition to $L^{+}(u)$ and $L^{-}(u)$ introduce matrices

$$
F^{ \pm}(u)=\left[\begin{array}{cccc}
1 & 0 & \ldots & 0 \\
f_{21}^{ \pm}(u) & 1 & \ldots & 0 \\
\vdots & \vdots & \ddots & \vdots \\
f_{N 1}^{ \pm}(u) & f_{N 2}^{ \pm}(u) & \ldots & 1
\end{array}\right], \quad E^{ \pm}(u)=\left[\begin{array}{cccc}
1 & e_{12}^{ \pm}(u) & \ldots & e_{1 N}^{ \pm}(u) \\
0 & 1 & \ldots & e_{2 N}^{ \pm}(u) \\
\vdots & \vdots & \ddots & \vdots \\
0 & 0 & \ldots & 1
\end{array}\right]
$$

and $H^{ \pm}(u)=\operatorname{diag}\left[h_{1}^{ \pm}(u), \ldots, h_{N}^{ \pm}(u)\right]$, such that

$$
L^{ \pm}(u)=F^{ \pm}(u) H^{ \pm}(u) E^{ \pm}(u) \text {. }
$$

Their entries are found by the quasideterminant formulas [13]:

$$
h_{i}^{ \pm}(u)=\left|\begin{array}{cccc}
l_{11}^{ \pm}(u) & \ldots & l_{1 i-1}^{ \pm}(u) & l_{1 i}^{ \pm}(u) \\
\vdots & \ddots & \vdots & \vdots \\
l_{i-11}^{ \pm}(u) & \ldots & l_{i-1 i-1}^{ \pm}(u) & l_{i-1 i}^{ \pm}(u) \\
l_{i 1}^{ \pm}(u) & \ldots & l_{i i-1}^{ \pm}(u) & l_{i i}^{ \pm}(u)
\end{array}\right|, \quad i=1, \ldots, N
$$

whereas

$$
e_{i j}^{ \pm}(u)=h_{i}^{ \pm}(u)^{-1}\left|\begin{array}{cccc}
l_{11}^{ \pm}(u) & \ldots & l_{1 i-1}^{ \pm}(u) & l_{1 j}^{ \pm}(u) \\
\vdots & \ddots & \vdots & \vdots \\
l_{i-11}^{ \pm}(u) & \ldots & l_{i-1 i-1}^{ \pm}(u) & l_{i-1 j}^{ \pm}(u) \\
l_{i 1}^{ \pm}(u) & \ldots & l_{i i-1}^{ \pm}(u) & l_{i j}^{ \pm}(u)
\end{array}\right|
$$

and

$$
f_{j i}^{ \pm}(u)=\left|\begin{array}{cccc}
l_{11}^{ \pm}(u) & \ldots & l_{1 i-1}^{ \pm}(u) & l_{1 i}^{ \pm}(u) \\
\vdots & \ddots & \vdots & \vdots \\
l_{i-11}^{ \pm}(u) & \ldots & l_{i-1 i-1}^{ \pm}(u) & l_{i-1 i}^{ \pm}(u) \\
l_{j 1}^{ \pm}(u) & \ldots & l_{j i-1}^{ \pm}(u) & l_{j i}^{ \pm}(u)
\end{array}\right| h_{i}^{ \pm}(u)^{-1}
$$

for $1 \leqslant i<j \leqslant N$. Set

$$
X_{i}^{+}(u)=e_{i, i+1}^{+}(u)-e_{i, i+1}^{-}(u), \quad X_{i}^{-}(u)=f_{i+1, i}^{+}(u)-f_{i+1, i}^{-}(u),
$$

for $i=1, \ldots, n-1$, and

$$
\begin{aligned}
& X_{n}^{+}(u)= \begin{cases}e_{n, n+1}^{+}(u)-e_{n, n+1}^{-}(u) & \text { for types } B_{n} \text { and } C_{n}, \\
e_{n-1, n+1}^{+}(u)-e_{n-1, n+1}^{-}(u) & \text { for type } D_{n},\end{cases} \\
& X_{n}^{-}(u)= \begin{cases}f_{n+1, n}^{+}(u)-f_{n+1, n}^{-}(u) & \text { for types } B_{n} \text { and } C_{n}, \\
f_{n+1, n-1}^{+}(u)-f_{n+1, n-1}^{-}(u) & \text { for type } D_{n} .\end{cases}
\end{aligned}
$$

Combine the generators $x_{i, m}^{ \pm}$of the algebra $\mathrm{U}_{q}(\widehat{\mathfrak{g}})$ into the series

$$
x_{i}^{ \pm}(u)=\sum_{m \in \mathbb{Z}} x_{i, m}^{ \pm} u^{-m}
$$


By the Main Theorems of [20] and [21], the maps

$$
\begin{aligned}
& x_{i}^{ \pm}(u) \mapsto\left(q_{i}-q_{i}^{-1}\right)^{-1} X_{i}^{ \pm}\left(u q^{i}\right), \\
& \psi_{i}(u) \mapsto h_{i+1}^{-}\left(u q^{i}\right) h_{i}^{-}\left(u q^{i}\right)^{-1}, \\
& \varphi_{i}(u) \mapsto h_{i+1}^{+}\left(u q^{i}\right) h_{i}^{+}\left(u q^{i}\right)^{-1},
\end{aligned}
$$

for $i=1, \ldots, n-1$, and

$$
\begin{gathered}
x_{n}^{ \pm}(u) \mapsto \begin{cases}\left(q_{n}-q_{n}^{-1}\right)^{-1}[2]_{q_{n}}^{-1 / 2} X_{n}^{ \pm}\left(u q^{n}\right) & \text { for type } B_{n}, \\
\left(q_{n}-q_{n}^{-1}\right)^{-1} X_{n}^{ \pm}\left(u q^{n+1}\right) & \text { for type } C_{n}, \\
\left(q_{n}-q_{n}^{-1}\right)^{-1} X_{n}^{ \pm}\left(u q^{n-1}\right) & \text { for type } D_{n},\end{cases} \\
\psi_{n}(u) \mapsto \begin{cases}h_{n+1}^{-}\left(u q^{n}\right) h_{n}^{-}\left(u q^{n}\right)^{-1} & \text { for type } B_{n}, \\
h_{n+1}^{-}\left(u q^{n+1}\right) h_{n}^{-}\left(u q^{n+1}\right)^{-1} & \text { for type } C_{n}, \\
h_{n+1}^{-}\left(u q^{n-1}\right) h_{n-1}^{-}\left(u q^{n-1}\right)^{-1} & \text { for type } D_{n},\end{cases} \\
\varphi_{n}(u) \mapsto \begin{cases}h_{n+1}^{+}\left(u q^{n}\right) h_{n}^{+}\left(u q^{n}\right)^{-1} & \text { for type } B_{n}, \\
h_{n+1}^{+}\left(u q^{n+1}\right) h_{n}^{+}\left(u q^{n+1}\right)^{-1} & \text { for type } C_{n}, \\
h_{n+1}^{+}\left(u q^{n-1}\right) h_{n-1}^{+}\left(u q^{n-1}\right)^{-1} & \text { for type } D_{n},\end{cases}
\end{gathered}
$$

define an isomorphism $\mathrm{U}_{q}(\widehat{\mathfrak{g}}) \rightarrow \mathrm{U}_{q}^{R}(\widehat{\mathfrak{g}})$.

\subsubsection{Central elements of the extended quantum affine algebra}

The extended quantum affine algebra is defined by the same presentation as the algebra $\mathrm{U}_{q}^{R}(\widehat{\mathfrak{g}})$, except that the relation (3.10) is omitted. It was shown in [20,21], that this algebra can be explicitly described in terms of the Gaussian generators by producing complete sets of relations. We will denote the extended algebra by $\mathrm{U}_{q}^{\text {ext }}(\widehat{\mathfrak{g}})$ and identify its $R$-matrix and Gaussian presentations.

The maps described above can be understood as an embedding $\iota: \mathrm{U}_{q}(\widehat{\mathfrak{g}}) \hookrightarrow \mathrm{U}_{q}^{\text {ext }}(\widehat{\mathfrak{g}})$ so that we can regard $\mathrm{U}_{q}(\widehat{\mathfrak{g}})$ as a subalgebra of $\mathrm{U}_{q}^{\text {ext }}(\widehat{\mathfrak{g}})$. This subalgebra can also be described with the use of the multiplication automorphisms

$$
\mu_{f}: \quad \mathrm{U}_{q}^{\operatorname{ext}}(\widehat{\mathfrak{g}}) \rightarrow \mathrm{U}_{q}^{\operatorname{ext}}(\widehat{\mathfrak{g}}), \quad L^{ \pm}(u) \mapsto f^{ \pm}(u) L^{ \pm}(u),
$$

where

$$
f^{ \pm}(u)=\sum_{m=0}^{\infty} f^{ \pm}[\mp m] u^{ \pm m}, \quad f^{ \pm}[\mp m] \in \mathbb{C}, \quad f^{+}[0] f^{-}[0]=1 .
$$

Namely, the image of $\mathrm{U}_{q}(\widehat{\mathfrak{g}})$ under the embedding $\iota$ consists of the elements in $\mathrm{U}_{q}^{\text {ext }}(\widehat{\mathfrak{g}})$ which are fixed by all automorphisms of the form (3.15).

All coefficients of the series $z^{ \pm}(u)$ given by

$$
z^{ \pm}(u)= \begin{cases}\prod_{i=1}^{n} h_{i}^{ \pm}\left(u \xi q^{2 i}\right)^{-1} \prod_{i=1}^{n} h_{i}^{ \pm}\left(u \xi q^{2 i-2}\right) \cdot h_{n+1}^{ \pm}(u) h_{n+1}^{ \pm}(u q) & \text { for } N=2 n+1, \\ \prod_{i=1}^{n-1} h_{i}^{ \pm}\left(u \xi q^{2 i}\right)^{-1} \prod_{i=1}^{n} h_{i}^{ \pm}\left(u \xi q^{2 i-2}\right) \cdot h_{n+1}^{ \pm}(u) & \text { for } N=2 n\end{cases}
$$

belong to the center of the algebra $\mathrm{U}_{q}^{\text {ext }}(\widehat{\mathfrak{g}})$. If $N=2 n$ then the constant terms of the series $z^{ \pm}(u)$ are the central elements $z^{ \pm}[0]=l_{n n}^{ \pm}[0] l_{n^{\prime} n^{\prime}}^{ \pm}[0]$. In this case we will extend this algebra 
by adjoining the square roots $z^{ \pm}[0]^{1 / 2}$. Then in all three cases there exist power series $\zeta^{ \pm}(u)$ with coefficients in the center of $\mathrm{U}_{q}^{\operatorname{ext}}(\widehat{\mathfrak{g}})$ such that $\zeta^{ \pm}(u) \zeta^{ \pm}(u \xi)=z^{ \pm}(u)$. Under the automorphism (3.15) we have

$$
\mu_{f}: \zeta^{ \pm}(u) \mapsto f^{ \pm}(u) \zeta^{ \pm}(u) .
$$

This implies that the coefficients of the entries of the matrices $\zeta^{ \pm}(u)^{-1} L^{ \pm}(u)$ belong to the subalgebra $\mathrm{U}_{q}(\widehat{\mathfrak{g}}) \subset \mathrm{U}_{q}^{\text {ext }}(\widehat{\mathfrak{g}})$. Therefore, if $\mathcal{C}$ denotes the subalgebra of $\mathrm{U}_{q}^{\text {ext }}(\widehat{\mathfrak{g}})$ generated by the coefficients of the series $\zeta^{ \pm}(u)$, then we have the tensor product decomposition

$$
\mathrm{U}_{q}^{\text {ext }}(\widehat{\mathfrak{g}})=\mathrm{U}_{q}(\widehat{\mathfrak{g}}) \otimes \mathcal{C}
$$

assuming that the algebra $\mathrm{U}_{q}(\widehat{\mathfrak{g}})$ is extended by adjoining square roots in types $C_{n}$ and $D_{n}$ as in Remark 3.1. In the algebra $\mathrm{U}_{q}^{\text {ext }}(\widehat{\mathfrak{g}})$ we have

$$
L^{ \pm}(u) D L^{ \pm}(u \xi)^{\mathrm{t}} D^{-1}=z^{ \pm}(u),
$$

so that the subalgebra $\mathrm{U}_{q}(\widehat{\mathfrak{g}})$ can also be regarded as the quotient of $\mathrm{U}_{q}^{\text {ext }}(\widehat{\mathfrak{g}})$ by the relations $z^{ \pm}(u)=1$. The following relations are implied by (3.17), and they were essentially derived in Section 4.5 of [20] and [21].

Lemma 3.2. In the algebra $\mathrm{U}_{q}^{\operatorname{ext}}(\widehat{\mathfrak{g}})$ we have

$$
\begin{aligned}
& h_{1}^{ \pm}(u \xi) h_{1^{\prime}}^{ \pm}(u)=z^{ \pm}(u), \\
& h_{i}^{ \pm}\left(u \xi q^{2 i}\right) h_{i^{\prime}}^{ \pm}(u)=h_{i+1}^{ \pm}\left(u \xi q^{2 i}\right) h_{(i+1)^{\prime}}^{ \pm}(u),
\end{aligned}
$$

where $i=1, \ldots, n$ for $N=2 n+1$, and $i=1, \ldots, n-1$ for $N=2 n$.

Remark 3.3. Note that for the parameters $d_{i j}(u, v)$ defined in $(3.3)$ we have $d_{j^{\prime} i^{\prime}}(u, v)=$ $d_{i j}(u, v)$. Therefore the $R$-matrix (3.2) possesses the symmetry property

$$
R^{T_{1} T_{2}}(u, v)=R_{21}(u, v),
$$

where $R_{21}(u, v)=P R(u, v) P$, while $T$ denotes the standard matrix transposition with $e_{i j}^{T}=e_{j i}$ and $T_{a}$ is the partial transposition applied to the $a$-th copy of the endomorphism algebra End $\mathbb{C}^{N}$. We can use the $R$-matrix $R_{21}(u, v)$ instead of $R(u, v)$ to define the extended quantum affine algebra $\widetilde{\mathrm{U}}_{q}^{\text {ext }}(\widehat{\mathfrak{g}})$ in a way similar to $\mathrm{U}_{q}^{\text {ext }}(\widehat{\mathfrak{g}})$, by using the relations

$$
\begin{aligned}
& R_{21}(u, v) \widetilde{L}_{1}^{ \pm}(u) \widetilde{L}_{2}^{ \pm}(v)=\widetilde{L}_{2}^{ \pm}(v) \widetilde{L}_{1}^{ \pm}(u) R_{21}(u, v), \\
& R_{21}(u, v) \widetilde{L}_{1}^{+}(u) \widetilde{L}_{2}^{-}(v)=\widetilde{L}_{2}^{-}(v) \widetilde{L}_{1}^{+}(u) R_{21}(u, v),
\end{aligned}
$$

where we impose the opposite zero mode conditions

$$
\widetilde{l}_{i j}^{+}[0]=\widetilde{l}_{j i}^{-}[0]=0 \quad \text { for } \quad i>j \quad \text { and } \quad \widetilde{l}_{i i}^{+}[0] \widetilde{l}_{i i}^{-}[0]=\widetilde{l}_{i i}^{-}[0] \widetilde{l}_{i i}^{+}[0]=1 .
$$

The symmetry property (3.18) implies that the mapping

$$
\widetilde{L}^{ \pm}(u) \mapsto L^{ \pm}(u)^{T}
$$

defines an anti-isomorphism $\widetilde{\mathrm{U}}_{q}^{\text {ext }}(\widehat{\mathfrak{g}}) \rightarrow \mathrm{U}_{q}^{\text {ext }}(\widehat{\mathfrak{g}})$. Using the definition of quasideterminants, we obtain the following formulas for the images of the respective Gaussian generators

$$
\widetilde{h}_{i}^{ \pm}(u) \mapsto h_{i}^{ \pm}(u), \quad \widetilde{e}_{i j}^{ \pm}(u) \mapsto f_{j i}^{ \pm}(u) \quad \text { and } \quad \widetilde{f}_{i j}^{ \pm}(u) \mapsto e_{j i}^{ \pm}(u) .
$$




\subsubsection{Hopf algebra structure}

The quantum affine algebra $\mathrm{U}_{q}^{\text {ext }}(\widehat{\mathfrak{g}})$ possesses a Hopf algebra structure defined by the coproduct

$$
\Delta: l_{i j}^{ \pm}(u) \mapsto \sum_{k=1}^{N} l_{i k}^{ \pm}(u) \otimes l_{k j}^{ \pm}(u),
$$

the antipode

$$
S: \quad L^{ \pm}(u) \mapsto L^{ \pm}(u)^{-1}
$$

and the counit

$$
\varepsilon: L^{ \pm}(u) \mapsto 1 .
$$

Proposition 3.4. In the Hopf algebra $\mathrm{U}_{q}^{\text {ext }}(\widehat{\mathfrak{g}})$ we have

$$
\Delta: z^{ \pm}(u) \mapsto z^{ \pm}(u) \otimes z^{ \pm}(u)
$$

and

$$
S: \quad z^{ \pm}(u) \mapsto z^{ \pm}(u)^{-1} .
$$

In particular, $\mathrm{U}_{q}(\widehat{\mathfrak{g}})$ is a Hopf subalgebra of $\mathrm{U}_{q}^{\text {ext }}(\widehat{\mathfrak{g}})$. Moreover,

$$
S^{2}: L^{ \pm}(u) \mapsto \frac{z^{ \pm}(u)}{z^{ \pm}(u \xi)} L^{ \pm}\left(u \xi^{2}\right) .
$$

Proof. The formulas for the images of the series $z^{ \pm}(u)$ under the maps $\Delta$ and $S$ follow easily from the definition of $z^{ \pm}(u)$ and the Hopf algebra axioms. For the proof of (3.23) use the relation

$$
L^{ \pm}(u)^{-1}=z^{ \pm}(u)^{-1} D L^{ \pm}(u \xi)^{\mathrm{t}} D^{-1}
$$

and apply (3.22). Furthermore, for the power series $\zeta^{ \pm}(u)$ we have

$$
\Delta: \zeta^{ \pm}(u) \mapsto \zeta^{ \pm}(u) \otimes \zeta^{ \pm}(u),
$$

and so

$$
\Delta\left(\mathrm{U}_{q}(\widehat{\mathfrak{g}})\right) \subset \mathrm{U}_{q}(\widehat{\mathfrak{g}}) \otimes \mathrm{U}_{q}(\widehat{\mathfrak{g}}), \quad S\left(\mathrm{U}_{q}(\widehat{\mathfrak{g}})\right) \subset \mathrm{U}_{q}(\widehat{\mathfrak{g}}),
$$

thus proving that $\mathrm{U}_{q}(\widehat{\mathfrak{g}})$ is a Hopf subalgebra of $\mathrm{U}_{q}^{\text {ext }}(\widehat{\mathfrak{g}})$.

\subsubsection{Consistency with the triangular decomposition}

Denote by $\mathrm{U}_{q}(\widehat{\mathfrak{g}})^{+}$(respectively, $\mathrm{U}_{q}(\widehat{\mathfrak{g}})^{-}$) the subalgebra of $\mathrm{U}_{q}(\widehat{\mathfrak{g}})$ generated by the elements $x_{i, m}^{+}$ (respectively, $x_{i, m}^{-}$), and denote by $\mathrm{U}_{q}(\widehat{\mathfrak{g}})^{0}$ the subalgebra generated by $k_{i}^{ \pm 1}$ and $a_{i, l}$ together with the additional elements in types $C_{n}$ and $D_{n}$, introduced in Remark 3.1. The multiplication map provides the triangular decomposition isomorphism

$$
\mathrm{U}_{q}(\widehat{\mathfrak{g}})^{-} \otimes \mathrm{U}_{q}(\widehat{\mathfrak{g}})^{0} \otimes \mathrm{U}_{q}(\widehat{\mathfrak{g}})^{+} \cong \mathrm{U}_{q}(\widehat{\mathfrak{g}})
$$

as proved in [4]; see also [16] for a generalization to quantum affinizations of symmetrizable Kac-Moody algebras.

Here we aim to establish a key property of the Gauss decomposition by showing that it is consistent with the triangular decomposition (see Proposition 3.8 below). We will rely on a few relations for the Gaussian generators described in the following lemmas.

We will use a standard notation $[x, y]_{q}=x y-q y x$ for $q$-commutators and begin by proving some $q$-commutator formulas; cf. [22, Lemma 5.6]. 
Lemma 3.5. For any $k<i<j<k^{\prime}$ such that $i \neq j^{\prime}$ we have

$$
\left[e_{k i}^{ \pm}(u), e_{i j}^{-}[0]\right]_{q}=\left(1-q^{2}\right) e_{k j}^{ \pm}(u) .
$$

Proof. Due to the consistency property of Gauss decompositions for subalgebras as stated in Proposition 4.2 in [20] and [21], we may assume without loss of generality, that $k=1$. By taking matrix entries in (3.8) and (3.9), write the defining relations in terms of the series $l_{i j}^{ \pm}(u)$ to get

$$
\begin{aligned}
\left(u q^{\delta_{i j}}\right. & \left.-v q^{-\delta_{i j}}\right) l_{i a}^{ \pm}(u) l_{j b}^{ \pm}(v)+\left(q-q^{-1}\right)\left(u \delta_{i<j}+v \delta_{i>j}\right) l_{j a}^{ \pm}(u) l_{i b}^{ \pm}(v) \\
& -\delta_{i j^{\prime}} \frac{u-v}{u-v \xi} \sum_{k=1}^{N} d_{j k}(u, v) l_{k^{\prime} a}^{ \pm}(u) l_{k b}^{ \pm}(v) \\
= & \left(u q^{\delta_{a b}}-v q^{-\delta_{a b}}\right) l_{j b}^{ \pm}(v) l_{i a}^{ \pm}(u)+\left(q-q^{-1}\right)\left(u \delta_{a>b}+v \delta_{a<b}\right) l_{j a}^{ \pm}(v) l_{i b}^{ \pm}(u) \\
& -\delta_{a b^{\prime}} \frac{u-v}{u-v \xi} \sum_{c=1}^{N} d_{c b}(u, v) l_{j c}^{ \pm}(v) l_{i c^{\prime}}^{ \pm}(u),
\end{aligned}
$$

and

$$
\begin{aligned}
\left(u q^{\delta_{i j}}\right. & \left.-v q^{-\delta_{i j}}\right) l_{i a}^{+}(u) l_{j b}^{-}(v)+\left(q-q^{-1}\right)\left(u \delta_{i<j}+v \delta_{i>j}\right) l_{j a}^{+}(u) l_{i b}^{-}(v) \\
& -\delta_{i j^{\prime}} \frac{u-v}{u-v \xi} \sum_{k=1}^{N} d_{j k}(u, v) l_{k^{\prime} a}^{+}(u) l_{k b}^{-}(v) \\
= & \left(u q^{\delta_{a b}}-v q^{-\delta_{a b}}\right) l_{j b}^{-}(v) l_{i a}^{+}(u)+\left(q-q^{-1}\right)\left(u \delta_{a>b}+v \delta_{a<b}\right) l_{j a}^{-}(v) l_{i b}^{+}(u) \\
& -\delta_{a b^{\prime}} \frac{u-v}{u-v \xi} \sum_{c=1}^{N} d_{c b}(u, v) l_{j c}^{-}(v) l_{i c^{\prime}}^{+}(u),
\end{aligned}
$$

where $d_{i j}(u, v)$ are defined in (3.3). If $1<i<j$ and $j \neq i^{\prime}$, then they give

$$
\begin{aligned}
& (u-v) l_{1 i}^{ \pm}(u) l_{i j}^{-}(v)+\left(q-q^{-1}\right) u l_{i i}^{ \pm}(u) l_{1 j}^{-}(v) \\
& \quad=(u-v) l_{i j}^{-}(v) l_{1 i}^{ \pm}(u)+\left(q-q^{-1}\right) v l_{i i}^{-}(v) l_{1 j}^{ \pm}(u) .
\end{aligned}
$$

By comparing the coefficients of $v$ on both sides, we come to the relations

$$
l_{1 i}^{ \pm}(u) l_{i j}^{-}[0]=l_{i j}^{-}[0] l_{1 i}^{ \pm}(u)-\left(q-q^{-1}\right) l_{i i}^{-}[0] l_{1 j}^{ \pm}(u) .
$$

Similarly, assuming that $1<i \leqslant m$ and $m \neq 1^{\prime}$, we get

$$
\begin{aligned}
& (u-v) l_{11}^{ \pm}(u) l_{i m}^{-}(v)+\left(q-q^{-1}\right) u l_{i 1}^{ \pm}(u) l_{1 m}^{-}(v) \\
& \quad=(u-v) l_{i m}^{-}(v) l_{11}^{ \pm}(u)+\left(q-q^{-1}\right) v l_{i 1}^{-}(v) l_{1 m}^{ \pm}(u),
\end{aligned}
$$

which yields

$$
l_{11}^{ \pm}(u) l_{i m}^{-}[0]=l_{i m}^{-}[0] l_{11}^{ \pm}(u) .
$$

Since $l_{1 i}^{ \pm}(u)=l_{11}^{ \pm}(u) e_{1 i}^{ \pm}(u)$, together with relation (3.24) this implies

$$
e_{1 i}^{ \pm}(u) l_{i j}^{-}[0]=l_{i j}^{-}[0] e_{1 i}^{ \pm}(u)-\left(q-q^{-1}\right) l_{i i}^{-}[0] e_{1 j}^{ \pm}(u) .
$$

Applying the defining relations again, for any $1<i<1^{\prime}$ we get

$$
(u-v) l_{1 i}^{ \pm}(u) l_{i i}^{-}(v)+\left(q-q^{-1}\right) u l_{i i}^{ \pm}(u) l_{1 i}^{-}(v)=\left(u q-v q^{-1}\right) l_{i i}^{-}(v) l_{1 i}^{ \pm}(u) .
$$


Comparing the coefficients of $v$ on both sides, we come to

$$
l_{1 i}^{ \pm}(u) l_{i i}^{-}[0]=q^{-1} l_{i i}^{-}[0] l_{1 i}^{ \pm}(u)
$$

which implies

$$
e_{1 i}^{ \pm}(u) l_{i i}^{-}[0]=q^{-1} l_{i i}^{-}[0] e_{1 i}^{ \pm}(u) .
$$

Hence, using this together with the decomposition $l_{i j}^{-}[0]=l_{i i}^{-}[0] e_{i j}^{-}[0]$, we derive from (3.26) that

$$
q^{-1} e_{1 i}^{ \pm}(u) e_{i j}^{-}[0]=e_{i j}^{-}[0] e_{1 i}^{ \pm}(u)-\left(q-q^{-1}\right) e_{1 j}^{ \pm}(u) .
$$

We can write this as the $q$-commutator relation

$$
\left[e_{1 i}^{ \pm}(u), e_{i j}^{-}[0]\right]_{q}=\left(1-q^{2}\right) e_{1 j}^{ \pm}(u)
$$

as required.

Lemma 3.6. For $n \geqslant 2$ we have

$$
\left[e_{12^{\prime}}^{ \pm}(u), e_{2^{\prime} 1^{\prime}}^{-}[0]\right]=\left(1-q^{2}\right)\left(e_{11^{\prime}}^{ \pm}(u)+e_{12}^{ \pm}(u) e_{12^{\prime}}^{ \pm}(u)\right) .
$$

Proof. The defining relations give

$$
\begin{aligned}
& (u-v) l_{11}^{ \pm}(u) l_{2^{\prime} 1^{\prime}}^{-}(v)+\left(q-q^{-1}\right) u l_{2^{\prime} 1}^{ \pm}(u) l_{11^{\prime}}^{-}(v) \\
& =(u-v) l_{2^{\prime} 1^{\prime}}^{-}(v) l_{11}^{ \pm}(u)+\left(q-q^{-1}\right) v l_{2^{\prime} 1}^{-}(v) l_{11^{\prime}}^{ \pm}(u)-\frac{u-v}{u-v \xi} \sum_{c=1}^{N} d_{c 1^{\prime}}(u, v) l_{2^{\prime} c}^{-}(v) l_{1 c^{\prime}}^{ \pm}(u) .
\end{aligned}
$$

By comparing the coefficients of $v$ on both sides we come to the relation

$$
l_{2^{\prime} 1^{\prime}}^{-}[0] l_{11}^{ \pm}(u)=q^{-1} l_{11}^{ \pm}(u) l_{2^{\prime} 1^{\prime}}^{-}[0]-\left(q-q^{-1}\right) l_{2^{\prime} 2^{\prime}}^{-}[0] l_{12}^{ \pm}(u) .
$$

On the other hand, taking $i=2^{\prime}$ and $j=1^{\prime}$ in (3.24) we get

$$
l_{12^{\prime}}^{ \pm}(u) l_{2^{\prime} 1^{\prime}}^{-}[0]=l_{2^{\prime} 1^{\prime}}^{-}[0] l_{12^{\prime}}^{ \pm}(u)-\left(q-q^{-1}\right) l_{2^{\prime} 2^{\prime}}^{-}[0] l_{11^{\prime}}^{ \pm}(u) .
$$

Write $l_{1 i}^{ \pm}(u)=l_{11}^{ \pm}(u) e_{1 i}^{ \pm}(u)$ for $i=1^{\prime}, 2^{\prime}$ and use (3.28) together with (3.25) to bring this to the form

$$
e_{12^{\prime}}^{ \pm}(u) l_{2^{\prime} 1^{\prime}}^{-}[0]=q^{-1} l_{2^{\prime} 1^{\prime}}^{-}[0] e_{12^{\prime}}^{ \pm}(u)-\left(q-q^{-1}\right) l_{2^{\prime} 2^{\prime}}^{-}[0]\left(e_{11^{\prime}}^{ \pm}(u)+e_{12}^{ \pm}(u) e_{12^{\prime}}^{ \pm}(u)\right) .
$$

Finally, write $l_{2^{\prime} 1^{\prime}}^{-}[0]=l_{2^{\prime} 2^{\prime}}^{-}[0] e_{2^{\prime} 1^{\prime}}^{-}[0]$ and apply (3.27) with $i=2^{\prime}$.

Lemma 3.7. For any $i<j<i^{\prime}$ we have the relations

$$
e_{i j}^{ \pm}\left(u \xi q^{2 i}\right)=q^{\bar{\jmath}-\bar{\imath}+1} \sum_{s=0}^{j-i-1}(-1)^{s+1} \sum_{j^{\prime}=a_{0}<a_{1}<\cdots<a_{s+1}=i^{\prime}} e_{a_{0} a_{1}}^{ \pm}(u) e_{a_{1} a_{2}}^{ \pm}(u) \cdots e_{a_{s} a_{s+1}}^{ \pm}(u) .
$$

Proof. We will rely on Proposition 4.2 in [20] and [21], which allows us to reduce the proof to the case $i=1$. Working in the algebra $\mathrm{U}_{q}^{\text {ext }}(\widehat{\mathfrak{g}})$, write $(3.17)$ in the form

$$
D L^{ \pm}(u \xi)^{\mathrm{t}} D^{-1}=L^{ \pm}(u)^{-1} z^{ \pm}(u)
$$

and take the $\left(j^{\prime}, 1^{\prime}\right)$ entries on both sides. Using the Gauss decompositions (3.11) and

$$
L^{ \pm}(u)^{-1}=E^{ \pm}(u)^{-1} H^{ \pm}(u)^{-1} F^{ \pm}(u)^{-1},
$$


we obtain

$$
q^{\overline{1}-\bar{\jmath}} h_{1}^{ \pm}(u \xi) e_{1 j}^{ \pm}(u \xi)=\widehat{e}_{j^{\prime} 1^{\prime}}^{ \pm}(u) h_{1^{\prime}}^{ \pm}(u)^{-1} z^{ \pm}(u),
$$

where we used the notation $\widehat{e}_{i j}^{ \pm}(u)$ for the $(i, j)$ entry of the matrix $E^{ \pm}(u)^{-1}$. Now apply (3.2) to replace $h_{1^{\prime}}^{ \pm}(u)^{-1} z^{ \pm}(u)$ with $h_{1}^{ \pm}(u \xi)$ and note that

$$
h_{1}^{ \pm}(u \xi) e_{1 j}^{ \pm}(u \xi)=q^{-1} e_{1 j}^{ \pm}\left(u \xi q^{2}\right) h_{1}^{ \pm}(u \xi) .
$$

Indeed, by the defining relations,

$$
\left(u q-v q^{-1}\right) l_{11}^{ \pm}(u) l_{1 j}^{ \pm}(v)=(u-v) l_{1 j}^{ \pm}(v) l_{11}^{ \pm}(u)+\left(q-q^{-1}\right) v l_{11}^{ \pm}(v) l_{1 j}^{ \pm}(u) .
$$

By setting $v=u q^{2}$ we get

$$
l_{1 j}^{ \pm}\left(u q^{2}\right) l_{11}^{ \pm}(u)=q l_{11}^{ \pm}\left(u q^{2}\right) l_{1 j}^{ \pm}(u) .
$$

Since $l_{1 j}^{ \pm}(u)=h_{1}^{ \pm}(u) e_{1 j}^{ \pm}(u),(3.29)$ follows. It remains to apply the formula

$$
\widehat{e}_{j^{\prime} 1^{\prime}}^{ \pm}(u)=\sum_{s=0}^{j-2}(-1)^{s+1} \sum_{j^{\prime}=a_{0}<a_{1}<\cdots<a_{s+1}=1^{\prime}} e_{a_{0} a_{1}}^{ \pm}(u) e_{a_{1} a_{2}}^{ \pm}(u) \cdots e_{a_{s} a_{s+1}}^{ \pm}(u)
$$

for the $\left(j^{\prime}, 1^{\prime}\right)$ entries of the inverse matrix $E^{ \pm}(u)^{-1}$.

Proposition 3.8. The images of the coefficients of all series $e_{i j}^{ \pm}(u)$ (respectively, $f_{j i}^{ \pm}(u)$ ) with $1 \leqslant i<j \leqslant N$ under the isomorphism $\mathrm{U}_{q}^{R}(\widehat{\mathfrak{g}}) \rightarrow \mathrm{U}_{q}(\widehat{\mathfrak{g}})$ belong to the subalgebra $\mathrm{U}_{q}(\widehat{\mathfrak{g}})^{+}$(respectively, $\left.\mathrm{U}_{q}(\widehat{\mathfrak{g}})^{-}\right)$. The images of the coefficients of all series $h_{i}^{ \pm}(u)$ with $i=1, \ldots, N$ belong to the subalgebra $\mathrm{U}_{q}(\widehat{\mathfrak{g}})^{0}$.

Proof. We will identify the coefficients of all the series with their images under the isomorphism $\mathrm{U}_{q}^{R}(\widehat{\mathfrak{g}}) \rightarrow \mathrm{U}_{q}(\widehat{\mathfrak{g}})$. We start by verifying the claim for the series $h_{i}^{+}(u)$; the argument for $h_{i}^{-}(u)$ is exactly the same. We have

$$
h_{i}^{+}(u)=\varphi_{1}\left(u q^{-1}\right) \varphi_{2}\left(u q^{-2}\right) \cdots \varphi_{i-1}\left(u q^{-i+1}\right) h_{1}^{+}(u), \quad i=2, \ldots, n .
$$

This relation is also valid for $i=n+1$ in type $B_{n}$, whereas

$$
h_{n+1}^{+}(u)=\varphi_{n}\left(u q^{-n-1}\right) h_{n}^{+}(u) \quad \text { and } \quad h_{n+1}^{+}(u)=\varphi_{n}\left(u q^{-n+1}\right) h_{n-1}^{+}(u)
$$

for types $C_{n}$ and $D_{n}$, respectively. Now substitute these expressions into the formula for the series $z^{+}(u)$ given in Section 3.2.2, so that the relation $z^{+}(u)=1$ would give an equation for the coefficients of the series $h_{1}^{+}(u)$. For type $B_{n}$ it reads

$$
h_{1}^{+}(u) h_{1}^{+}(u \xi) \prod_{i=1}^{n} \varphi_{i}\left(u q^{-i}\right) \varphi_{i}\left(u \xi q^{i}\right)=1 .
$$

Hence, the constant term $h_{1,0}^{+}$of $h_{1}^{+}(u)$ is found from $h_{1,0}^{+2}=k_{1}^{2} \cdots k_{n}^{2}$ which together with Lemma 3.2 implies that all coefficients of the series $h_{i}^{+}(u)$ with $i=1, \ldots, N$ belong to the subalgebra $\mathrm{U}_{q}(\widehat{\mathfrak{g}})^{0}$. The same conclusion is reached for types $C_{n}$ and $D_{n}$ from the respective formulas

$$
h_{1}^{+}(u) h_{1}^{+}(u \xi) \prod_{i=1}^{n-1} \varphi_{i}\left(u q^{-i}\right) \varphi_{i}\left(u \xi q^{i}\right) \cdot \varphi_{n}\left(u q^{-n-1}\right)=1
$$


and

$$
h_{1}^{+}(u) h_{1}^{+}(u \xi) \prod_{i=1}^{n-2} \varphi_{i}\left(u q^{-i}\right) \varphi_{i}\left(u \xi q^{i}\right) \cdot \varphi_{n-1}\left(u q^{-n+1}\right) \varphi_{n}\left(u \xi q^{n-1}\right)=1,
$$

which imply the relations $h_{1,0}^{+2}=k_{1}^{2} \cdots k_{n-1}^{2} k_{n}$ and $h_{1,0}^{+2}=k_{1}^{2} \cdots k_{n-2}^{2} k_{n-1} k_{n}$, respectively, for the constant terms.

Now turn to the series $e_{i j}^{ \pm}(u)$ and use induction on $n$. By Proposition 4.2 in [20] and [21], the coefficients of these series with $2 \leqslant i<j \leqslant 2^{\prime}$ can be regarded as elements of the algebra $\mathrm{U}_{q}\left(\widehat{\mathfrak{g}^{\prime}}\right)$ associated with the Lie algebra $\mathfrak{g}^{\prime}$ of rank $n-1$, which belongs to the same type as $\mathfrak{g}$. These coefficients coincide with the generators obtained from the Gauss decompositions of the associated $L$-operators, so that we can apply the induction hypothesis to suppose that the coefficients of all series $e_{i j}^{ \pm}(u)$ with $2 \leqslant i<j \leqslant 2^{\prime}$ belong to the subalgebra $\mathrm{U}_{q}(\widehat{\mathfrak{g}})^{+}$.

On the other hand, the coefficients of the series $e_{12}^{ \pm}(u)$ also belong to the subalgebra $\mathrm{U}_{q}(\widehat{\mathfrak{g}})^{+}$, and so by applying Lemma 3.5 we derive that the coefficients of the series $e_{1 j}^{ \pm}(u)$ with $j=2, \ldots, 2^{\prime}$ belong to $\mathrm{U}_{q}(\widehat{\mathfrak{g}})^{+}$, assuming that $n \geqslant 2$ for type $B_{n}$, and $n \geqslant 3$ for types $C_{n}$ and $D_{n}$. Furthermore, by Lemma 3.7 we have $e_{2^{\prime} 1^{\prime}}^{-}[0]=-e_{12}^{-}[0]$ so that applying Lemma 3.6 we may conclude that the required property is shared by the series $e_{11^{\prime}}^{ \pm}(u)$. The induction step is completed by another application of Lemma 3.7, which implies that the coefficients of the series $e_{j^{\prime} 1^{\prime}}^{ \pm}(u)$ with $j=2, \ldots, 2^{\prime}$ also belong to $\mathrm{U}_{q}(\widehat{\mathfrak{g}})^{+}$.

It remains to verify the induction base for the Lie algebras $\mathfrak{g}=\mathfrak{o}_{3}, \mathfrak{s p}_{4}$ and $\mathfrak{o}_{4}$. In the case $\mathfrak{g}=\mathfrak{o}_{3}$ it follows from the identity

$$
e_{12}^{ \pm}(u)^{2}=-\left(q^{1 / 2}+q^{-1 / 2}\right) e_{11^{\prime}}^{ \pm}(u)
$$

which is a particular case of [21, Lemma 4.9] obtained by taking the residue at $u=q^{-2} v$ in the second formula. In the case $\mathfrak{g}=\mathfrak{s p}_{4}$ it is sufficient to verify the identity

$$
\left[e_{12}^{ \pm}(u), e_{22^{\prime}}^{-}[0]\right]_{q^{2}}=\left(1-q^{4}\right) e_{12^{\prime}}^{ \pm}(u) .
$$

By the defining relations,

$$
\begin{aligned}
& (u-v) l_{12}^{ \pm}(u) l_{22^{\prime}}^{-}(v)+\left(q-q^{-1}\right) u l_{22}^{ \pm}(u) l_{12^{\prime}}^{-}(v) \\
& =(u-v) l_{22^{\prime}}^{-}(v) l_{12}^{ \pm}(u)+\left(q-q^{-1}\right) v l_{22}^{-}(v) l_{12^{\prime}}^{ \pm}(u)-\frac{u-v}{u-v \xi} \sum_{c=1}^{4} d_{c 2^{\prime}}(u, v) l_{2 c}^{-}(v) l_{1 c^{\prime}}^{ \pm}(u) .
\end{aligned}
$$

Comparing the coefficients of $v$ on both sides we derive

$$
l_{12}^{ \pm}(u) l_{22^{\prime}}^{-}[0]=q l_{22^{\prime}}^{-}[0] l_{12}^{ \pm}(u)+\left(q^{-1}-q^{3}\right) l_{22}^{-}[0] l_{12^{\prime}}^{ \pm}(u) .
$$

Relation (3.30) now follows from (3.25) and (3.27).

The particular case $\mathfrak{g}=\mathfrak{o}_{4}$ was considered in [21, Section 4.4]; it was shown therein that $e_{22^{\prime}}^{ \pm}(u)=0$, which is sufficient to complete the argument in this case. These relations essentially refer to the algebra $\mathrm{U}_{q}^{R}(\widehat{\mathfrak{g}})$ with $\mathfrak{g}=\mathfrak{o}_{2}$, whose definition extends to the abelian Lie algebra $\mathfrak{o}_{2}$. In this case we have $\xi=1$ and the defining relations give ${ }^{4}$

$$
\left(u q-v q^{-1}\right) l_{11^{\prime}}^{ \pm}(u) l_{11}^{ \pm}(v)=\left(u q^{-1}-v q\right) l_{11}^{ \pm}(v) l_{11^{\prime}}^{ \pm}(u) .
$$

By setting $v=u q^{2}$ we derive that $l_{11^{\prime}}^{ \pm}(u)=0$ and hence $e_{11^{\prime}}^{ \pm}(u)=0$.

The argument for the series $f_{j i}^{ \pm}(u)$ is quite similar to that for $e_{i j}^{ \pm}(u)$. As an alternative approach, one can work with the quantum affine algebra defined with the $R$-matrix $R_{21}(u, v)$ used instead of $R(u, v)$, and modify the above calculations for the series $e_{i j}^{ \pm}(u)$ accordingly; then apply the anti-isomorphism introduced in Remark 3.3.

\footnotetext{
${ }^{4}$ This corrects the formula used in the proof of [21, Lemma 4.13].
} 


\subsection{Highest weight representations}

Definition 3.9. A representation $V$ of the algebra $\mathrm{U}_{q}^{R}(\widehat{\mathfrak{g}})$ (or the extended quantum affine algebra $\left.\mathrm{U}_{q}^{\text {ext }}(\widehat{\mathfrak{g}})\right)$ is called a highest weight representation if there exists a nonzero vector $\zeta \in V$ such that $V$ is generated by $\zeta$ and the following relations hold:

$$
\begin{array}{ll}
l_{i j}^{ \pm}(u) \zeta=0 & \text { for } \quad 1 \leqslant i<j \leqslant N, \quad \text { and } \\
l_{i i}^{ \pm}(u) \zeta=\lambda_{i}^{ \pm}(u) \zeta & \text { for } \quad i=1, \ldots, N,
\end{array}
$$

for some formal series $\lambda_{i}^{+}(u) \in \mathbb{C}[[u]]$ and $\lambda_{i}^{-}(u) \in \mathbb{C}\left[\left[u^{-1}\right]\right]$. The vector $\zeta$ is called the highest vector of $V$.

Note that by (3.4), the product of the constant terms of $\lambda_{i}^{+}(u)$ and $\lambda_{i}^{-}(u)$ must equal 1 .

Proposition 3.10. In terms of the Gaussian generators, the conditions in Definition 3.9 are equivalent to the following:

$$
\begin{array}{ll}
e_{i j}^{ \pm}(u) \zeta=0 & \text { for } \quad 1 \leqslant i<j \leqslant N, \quad \text { and } \\
h_{i}^{ \pm}(u) \zeta=\lambda_{i}^{ \pm}(u) \zeta & \text { for } \quad i=1, \ldots, N .
\end{array}
$$

Proof. This is immediate from the Gauss decomposition formulas (3.11)-(3.13).

The algebra $\mathrm{U}_{q}^{R}(\widehat{\mathfrak{g}})$ (as well as $\mathrm{U}_{q}^{\text {ext }}(\widehat{\mathfrak{g}})$ ) admits a family of automorphisms

$$
L^{+}(u) \mapsto \mathcal{S} L^{+}(u) \quad \text { and } \quad L^{-}(u) \mapsto \mathcal{S}^{-1} L^{-}(u),
$$

parameterized by invertible diagonal matrices $\mathcal{S}=\operatorname{diag}\left[\sigma_{1}, \ldots, \sigma_{N}\right]$ satisfying the conditions

$$
\mathcal{S}^{\mathrm{t}}=\mathcal{S}^{-1}=\mathcal{S} \quad \text { or } \quad \mathcal{S}^{\mathrm{t}}=\mathcal{S}^{-1}=-\mathcal{S} .
$$

The second condition can occur only for $N=2 n$. When applied to the isomorphic algebra $\mathrm{U}_{q}(\widehat{\mathfrak{g}}) \cong \mathrm{U}_{q}^{R}(\widehat{\mathfrak{g}})$, the automorphisms (3.31) correspond to the sign automorphisms considered in [6, Section 12.2.B].

\section{Theorem 3.11.}

1. Any finite-dimensional irreducible representation of the algebra $\mathrm{U}_{q}^{R}(\widehat{\mathfrak{g}})$ is a highest weight representation. Up to twisting this representation with a suitable automorphism (3.31), its parameters satisfy the relations

$$
\frac{\lambda_{i}^{+}(u)}{\lambda_{i+1}^{+}(u)}=q^{\operatorname{deg} P_{i}} \frac{P_{i}\left(u q^{-2}\right)}{P_{i}(u)}=\frac{\lambda_{i}^{-}(u)}{\lambda_{i+1}^{-}(u)}, \quad i=1, \ldots, n-1,
$$

and

$$
\begin{array}{ll}
\frac{\lambda_{n}^{+}(u)}{\lambda_{n+1}^{+}(u)}=q^{\frac{1}{2} \operatorname{deg} P_{n}} \frac{P_{n}\left(u q^{-1}\right)}{P_{n}(u)}=\frac{\lambda_{n}^{-}(u)}{\lambda_{n+1}^{-}(u)} \quad \text { for type } B_{n}, \\
\frac{\lambda_{n}^{+}(u)}{\lambda_{n+1}^{+}(u)}=q^{2 \operatorname{deg} P_{n}} \frac{P_{n}\left(u q^{-4}\right)}{P_{n}(u)}=\frac{\lambda_{n}^{-}(u)}{\lambda_{n+1}^{-}(u)} \quad \text { for type } C_{n}, \\
\frac{\lambda_{n-1}^{+}(u)}{\lambda_{n+1}^{+}(u)}=q^{\operatorname{deg} P_{n}} \frac{P_{n}\left(u q^{-2}\right)}{P_{n}(u)}=\frac{\lambda_{n-1}^{-}(u)}{\lambda_{n+1}^{-}(u)} \quad \text { for type } D_{n},
\end{array}
$$

for some polynomials $P_{i}(u)$ in $u$, all with constant term 1 , where the first and second equalities are regarded in $\mathbb{C}[[u]]$ and $\mathbb{C}\left[\left[u^{-1}\right]\right]$, respectively. 
2. Every $n$-tuple $\left(P_{1}(u), \ldots, P_{n}(u)\right)$, where each $P_{i}(u)$ is a polynomial in $u$ with constant term 1 , arises in this way.

3. The series $\lambda_{i}^{ \pm}(u)$ satisfy the relations

$$
\lambda_{i}^{ \pm}\left(u \xi q^{2 i}\right) \lambda_{i^{\prime}}^{ \pm}(u)=\lambda_{i+1}^{ \pm}\left(u \xi q^{2 i}\right) \lambda_{(i+1)^{\prime}}^{ \pm}(u),
$$

where $i=0,1, \ldots, n$ for $N=2 n+1$, and $i=0,1, \ldots, n-1$ for $N=2 n$, and we set $\lambda_{0}^{ \pm}(u)=\lambda_{0^{\prime}}^{ \pm}(u):=1$.

Proof. Using the isomorphism $\mathrm{U}_{q}(\widehat{\mathfrak{g}}) \cong \mathrm{U}_{q}^{R}(\widehat{\mathfrak{g}})$ and the classification results recalled in Section 3.1, we find that any type $\mathbf{1}$ finite-dimensional irreducible representation $V$ of the algebra $\mathrm{U}_{q}(\widehat{\mathfrak{g}})$ is generated by a vector $\zeta$ such that

$$
\begin{array}{ll}
e_{i, i+1}^{ \pm}(u) \zeta=0 & \text { for } \quad i=1, \ldots, n, \\
h_{i}^{ \pm}(u) \zeta=\lambda_{i}^{ \pm}(u) \zeta & \text { for } \quad i=1, \ldots, n+1,
\end{array}
$$

for some formal series $\lambda_{i}^{+}(u) \in \mathbb{C}[[u]]$ and $\lambda_{i}^{-}(u) \in \mathbb{C}\left[\left[u^{-1}\right]\right]$, where for type $D_{n}$ relation (3.34) with $i=n$ should be replaced with $e_{n-1, n+1}^{ \pm}(u) \zeta=0$. Proposition 3.8 implies that $V$ is a highest weight representation of $\mathrm{U}_{q}^{R}(\widehat{\mathfrak{g}})$. Relations (3.32) follow from the results of [6, Section 12.2]. They can also be derived by considering a subalgebra $\mathrm{U}_{i} \subset \mathrm{U}_{q}^{R}(\widehat{\mathfrak{g}})$ associated with the $i$-th simple root of $\mathfrak{g}$ such that $\mathrm{U}_{i} \cong \mathrm{U}_{q}\left(\widehat{\mathfrak{s l}}_{2}\right)$. The cyclic span $\mathrm{U}_{i} \zeta$ is a finite-dimensional $\mathrm{U}_{q}\left(\widehat{\mathfrak{s l}}_{2}\right)$-module which yields the required conditions on the series $\lambda_{i}^{ \pm}(u)$; see [14, Section 3.1] for more details on this approach going back to [28].

Part 2 of the theorem follows from the classification results of [6, Section 12.2]. The proof relies on the Hopf algebra structure on $\mathrm{U}_{q}^{R}(\widehat{\mathfrak{g}})$ introduced in Section 3.2.3. It is implied by a well-known property of tensor products of representations. Namely, suppose that $V$ and $W$ are finite-dimensional irreducible representations of $\mathrm{U}_{q}^{R}(\widehat{\mathfrak{g}})$ with respective highest vectors $\zeta$ and $\eta$, with the parameter series $\left(\lambda_{1}^{ \pm}(u), \ldots, \lambda_{N}^{ \pm}(u)\right)$ and $\left(\mu_{1}^{ \pm}(u), \ldots, \mu_{N}^{ \pm}(u)\right)$. The coproduct formula (3.19) implies that the irreducible quotient $X$ of the cyclic span

$$
\mathrm{U}_{q}^{R}(\widehat{\mathfrak{g}})(\zeta \otimes \eta) \subset V \otimes W
$$

is a highest weight representation with the parameter series $\left(\lambda_{1}^{ \pm}(u) \mu_{1}^{ \pm}(u), \ldots, \lambda_{N}^{ \pm}(u) \mu_{N}^{ \pm}(u)\right)$. Therefore, if $V$ and $W$ are associated with the respective $n$-tuples of polynomials

$$
\left(P_{1}(u), \ldots, P_{n}(u)\right) \quad \text { and } \quad\left(Q_{1}(u), \ldots, Q_{n}(u)\right),
$$

then by the formulas of Part 1 , the representation $X$ is associated with the $n$-tuple

$$
\left(P_{1}(u) Q_{1}(u), \ldots, P_{n}(u) Q_{n}(u)\right) .
$$

Hence, to complete the proof of Part 2, one only needs to produce a finite-dimensional irreducible representation to each $n$-tuple of the form

$$
(1, \ldots, 1, a u+1,1, \ldots, 1), \quad a \in \mathbb{C} .
$$

The existence of such fundamental representations of the quantum affine algebra was established in [7]. Part 3 of the theorem is immediate from Lemma 3.2.

Corollary 3.12. All statements of Theorem 3.11 hold in the same form for the algebra $\mathrm{U}_{q}^{\text {ext }}(\widehat{\mathfrak{g}})$, except that the value $i=0$ is excluded for the conditions (3.33).

Proof. The arguments used in the proof of the theorem equally apply to the representations of the algebra $\mathrm{U}_{q}^{\text {ext }}(\widehat{\mathfrak{g}})$. Relation (3.33) with $i=0$ is now replaced by the property that the series $z^{ \pm}(u)$ acts in the highest weight representation as multiplication by $\lambda_{1}^{ \pm}(u \xi) \lambda_{1^{\prime}}^{ \pm}(u)$. 


\subsubsection{Representations of quantum affine algebras in type $A$}

Corresponding versions of the classification results described in Theorem 3.11 for the quantum affine algebras in type $A$ are well-known. They can be derived in the same way as for the types $B, C$ and $D$ from the isomorphism between the $R$-matrix and Drinfeld presentations of the quantum affine algebra $\mathrm{U}_{q}(\widehat{\mathfrak{g}})$ for $\mathfrak{g}=\mathfrak{s l}_{n}$ constructed in [8]. On the other hand, an independent proof of the classification theorem for the quantum affine algebras in the $R$-matrix presentation, which we state below, was given in [14] following the original approach of [28].

The quantum affine algebra $\mathrm{U}_{q}\left(\widehat{\mathfrak{s l}}_{n}\right)$ (with the trivial central charge) is defined by the formulas of Section 3.1, where the simple roots are chosen in the form (2.1). Finite-dimensional irreducible representations of $\mathrm{U}_{q}\left(\widehat{\mathfrak{s l}}_{n}\right)$ are described by the results recalled in that section.

Consider the $R$-matrix defined by

$$
R_{A}(u)=\sum_{i, j=1}^{n}\left(u q^{\delta_{i j}}-q^{-\delta_{i j}}\right) e_{i i} \otimes e_{j j}+\left(q-q^{-1}\right) \sum_{i, j=1}^{n}\left(u \delta_{i<j}+\delta_{i>j}\right) e_{i j} \otimes e_{j i} .
$$

The quantum affine algebra $\mathrm{U}_{q}^{R}\left(\widehat{\mathfrak{g l}}_{n}\right)$ (with the trivial central charge) is generated by elements $l_{i j}^{ \pm}[\mp m]$ with $1 \leqslant i, j \leqslant n$ and $m \in \mathbb{Z}_{+}$subject to the defining relations described in (3.4)-(3.9), where the parameter $N$ is replaced by $n$ and the $R$-matrix (3.1) is replaced by $R_{A}(u)$. The Hopf algebra structure on $\mathrm{U}_{q}^{R}\left(\widehat{\mathfrak{g l}}_{n}\right)$ is described by the maps (3.19), (3.20) and (3.21). The algebra $\mathrm{U}_{q}^{R}\left(\widehat{\mathfrak{s l}}_{n}\right)$ is a Hopf subalgebra of $\mathrm{U}_{q}^{R}\left(\widehat{\mathfrak{g l}}_{n}\right)$ which consists of all elements which are stable with respect to all automorphisms

$$
L^{ \pm}(u) \mapsto f^{ \pm}(u) L^{ \pm}(u)
$$

where $f^{ \pm}(u)$ are arbitrary series satisfying conditions (3.16). By the results of [8] the maps (3.14) define a Hopf algebra isomorphism $\mathrm{U}_{q}\left(\widehat{\mathfrak{s l}}_{n}\right) \rightarrow \mathrm{U}_{q}^{R}\left(\widehat{\mathfrak{s l}}_{n}\right)$, where the Gaussian generators are defined by (3.11). The algebra $\mathrm{U}_{q}^{R}\left(\widehat{\mathfrak{s l}}_{n}\right)$ (as well as $\mathrm{U}_{q}^{R}\left(\widehat{\mathfrak{g l}}_{n}\right)$ ) admits a family of automorphisms (3.31) parameterized by diagonal matrices $\mathcal{S}=\operatorname{diag}\left[\sigma_{1}, \ldots, \sigma_{n}\right]$ such that $\sigma_{i}= \pm 1$ for all $i$.

Highest weight representations of the algebra $\mathrm{U}_{q}^{R}\left(\widehat{\mathfrak{g l}}_{n}\right)$ are defined in the same way as in Definition 3.9. By restrictions, we get highest weight representations of the subalgebra $\mathrm{U}_{q}^{R}\left(\widehat{\mathfrak{s l}}_{n}\right)$. Proposition 3.10 holds in the same form. The following theorem is contained in [14, Theorem 3.6]. The proof of Theorem 3.11 applied to the quantum affine algebras of type $A$ provides another derivation of that result.

\section{Theorem 3.13.}

1. Any finite-dimensional irreducible representation of the algebra $\mathrm{U}_{q}^{R}\left(\widehat{\mathfrak{g l}}_{n}\right)$ (and $\left.\mathrm{U}_{q}^{R}\left(\widehat{\mathfrak{s l}}_{n}\right)\right)$ is a highest weight representation. Up to twisting this representation with a suitable automorphism (3.31), its parameters satisfy the relations

$$
\frac{\lambda_{i}^{+}(u)}{\lambda_{i+1}^{+}(u)}=q^{\operatorname{deg} P_{i}} \frac{P_{i}\left(u q^{-2}\right)}{P_{i}(u)}=\frac{\lambda_{i}^{-}(u)}{\lambda_{i+1}^{-}(u)}, \quad i=1, \ldots, n-1,
$$

for some polynomials $P_{i}(u)$ in $u$, all with constant term 1 , where the equalities are understood for the expansions of the rational function in $u$ as a series in $u$ and $u^{-1}$, respectively.

2. Every $n$-tuple of polynomials $\left(P_{1}(u), \ldots, P_{n-1}(u)\right)$ in $u$, where each $P_{i}(u)$ has constant term 1 , arises in this way. 


\subsubsection{Outlook}

The isomorphisms of $[20,21]$ do not rely on any Poincaré-Birkhoff-Witt-type theorem for the algebra $\mathrm{U}_{q}^{R}(\widehat{\mathfrak{g}})$. Therefore, such a theorem is implied by the results of Beck [4]. In particular, by Proposition 3.8, the image of the basis of the subalgebra $\mathrm{U}_{q}(\widehat{\mathfrak{g}})^{+}$under the isomorphism $\mathrm{U}_{q}(\widehat{\mathfrak{g}}) \rightarrow \mathrm{U}_{q}^{R}(\widehat{\mathfrak{g}})$ is a basis of the subalgebra $\mathrm{U}_{q}^{R}(\widehat{\mathfrak{g}})^{+}$generated by the coefficients of all series $e_{i j}^{ \pm}(u)$. However, a precise expression of the basis elements in terms of these coefficients is unknown.

Note also that the version of the Poincaré-Birkhoff-Witt theorem for the algebra $\mathrm{U}_{q}\left(\widehat{\mathfrak{g l}}_{N}\right)$ given in [14, Corollary 2.13] does not immediately extend to types $B, C$ and $D$, because of more complicated defining relations on the generators $l_{i j}^{ \pm}[m]$. It would be interesting to find an alternative form of the quadratic relations for the generator series to lead to such a version.

\section{A Modified isomorphism for Yangians}

Here we give a modified version of the isomorphism produced in [19, Main Theorem] which can be used to establish a different correspondence between the parameters of representations in the two realizations of the Yangians; cf. Section 2.2.1. An analogous isomorphism was used for type $A$ in [24, Section 3.1]; see also [5] for an isomorphism with an opposite presentation of the Yangian $\mathrm{Y}\left(\mathfrak{s l}_{N}\right)$. The new version is based on the alternative Gauss decomposition of the matrix $T(u)$, defined by

$$
T(u)=\bar{E}(u) \bar{H}(u) \bar{F}(u),
$$

where $\bar{E}(u), \bar{H}(u)$ and $\bar{F}(u)$ are uniquely determined matrices of the form

$$
\bar{E}(u)=\left[\begin{array}{cccc}
1 & \bar{e}_{12}(u) & \ldots & \bar{e}_{1 N}(u) \\
0 & 1 & \ldots & \bar{e}_{2 N}(u) \\
\vdots & \vdots & \ddots & \vdots \\
0 & 0 & \ldots & 1
\end{array}\right], \quad \bar{F}(u)=\left[\begin{array}{cccc}
1 & 0 & \ldots & 0 \\
\bar{f}_{21}(u) & 1 & \ldots & 0 \\
\vdots & \vdots & \ddots & \vdots \\
\bar{f}_{N 1}(u) & \bar{f}_{N 2}(u) & \ldots & 1
\end{array}\right]
$$

and $\bar{H}(u)=\operatorname{diag}\left[\bar{h}_{1}(u), \ldots, \bar{h}_{N}(u)\right]$. Define the series with coefficients in $\mathrm{Y}(\mathfrak{g})$ by

$$
\kappa_{i}(u)=\bar{h}_{i}(u+(i-1) / 2) \bar{h}_{i+1}(u+(i-1) / 2)^{-1}
$$

for $i=1, \ldots, n-1$, and

$$
\kappa_{n}(u)= \begin{cases}\bar{h}_{n}(u+(n-1) / 2) \bar{h}_{n+1}(u+(n-1) / 2)^{-1} & \text { for } \mathfrak{o}_{2 n+1}, \\ \bar{h}_{n}(u+n / 2) \bar{h}_{n+1}(u+n / 2)^{-1} & \text { for } \mathfrak{s p}_{2 n}, \\ \bar{h}_{n-1}(u+(n-2) / 2) \bar{h}_{n+1}(u+(n-2) / 2)^{-1} & \text { for } \mathfrak{o}_{2 n} .\end{cases}
$$

Furthermore, set

$$
\xi_{i}^{+}(u)=\bar{e}_{i+1}(u+(i-1) / 2), \quad \xi_{i}^{-}(u)=\bar{f}_{i+1 i}(u+(i-1) / 2)
$$

for $i=1, \ldots, n-1$,

$$
\xi_{n}^{+}(u)= \begin{cases}\bar{e}_{n n+1}(u+(n-1) / 2) & \text { for } \mathfrak{o}_{2 n+1} \\ \bar{e}_{n n+1}(u+n / 2) & \text { for } \mathfrak{s p}_{2 n} \\ \bar{e}_{n-1 n+1}(u+(n-2) / 2) & \text { for } \mathfrak{o}_{2 n}\end{cases}
$$


and

$$
\xi_{n}^{-}(u)= \begin{cases}\bar{f}_{n+1 n}(u+(n-1) / 2) & \text { for } \mathfrak{o}_{2 n+1}, \\ \frac{1}{2} \bar{f}_{n+1 n}(u+n / 2) & \text { for } \mathfrak{s p}_{2 n}, \\ \bar{f}_{n+1 n-1}(u+(n-2) / 2) & \text { for } \mathfrak{o}_{2 n} .\end{cases}
$$

Introduce elements of $\mathrm{Y}(\mathfrak{g})$ by the respective expansions into power series in $u^{-1}$,

$$
\kappa_{i}(u)=1+\sum_{r=0}^{\infty} \kappa_{i r} u^{-r-1} \quad \text { and } \quad \xi_{i}^{ \pm}(u)=\sum_{r=0}^{\infty} \xi_{i r}^{ \pm} u^{-r-1}
$$

for $i=1, \ldots, n$.

Theorem A.1. The mapping which sends the generators $\kappa_{i r}$ and $\xi_{i r}^{ \pm}$of $\mathrm{Y}^{D}(\mathfrak{g})$ to the elements of $\mathrm{Y}(\mathfrak{g})$ with the same names defines an isomorphism $\mathrm{Y}^{D}(\mathfrak{g}) \cong \mathrm{Y}(\mathfrak{g})$.

Proof. We will derive this result from [19, Main Theorem] as recalled in Section 2.2.1, by taking the composition of the isomorphism $\mathrm{Y}^{D}(\mathfrak{g}) \rightarrow \mathrm{Y}(\mathfrak{g})$ constructed therein, with certain automorphisms of the algebra $\mathrm{Y}(\mathfrak{g})$. We have the following quasideterminant formulas for the entries of the matrices $\bar{H}(u), \bar{E}(u)$ and $\bar{F}(u)[13]$ :

$$
\bar{h}_{i}(u)=\left|\begin{array}{cccc}
t_{i i}(u) & t_{i i+1}(u) & \ldots & t_{i N}(u) \\
t_{i+1 i}(u) & t_{i+1 i+1}(u) & \ldots & t_{i+1 N}(u) \\
\vdots & \vdots & \ddots & \vdots \\
t_{N i}(u) & t_{N i+1}(u) & \ldots & t_{N N}(u)
\end{array}\right|, \quad i=1, \ldots, N
$$

whereas

$$
\bar{e}_{i j}(u)=\left|\begin{array}{cccc}
t_{i j}(u) & t_{i j+1}(u) & \ldots & t_{i N}(u) \\
t_{j+1 j}(u) & t_{j+1 j+1}(u) & \ldots & t_{j+1 N}(u) \\
\vdots & \vdots & \ddots & \vdots \\
t_{N j}(u) & t_{N j+1}(u) & \ldots & t_{N N}(u)
\end{array}\right| h_{j}(u)^{-1}
$$

and

$$
\bar{f}_{j i}(u)=h_{j}(u)^{-1}\left|\begin{array}{cccc}
t_{j i}(u) & t_{j j+1}(u) & \ldots & t_{j N}(u) \\
t_{j+1 i}(u) & t_{j+1 j+1}(u) & \ldots & t_{j+1 N}(u) \\
\vdots & \vdots & \ddots & \vdots \\
t_{N i}(u) & t_{N j+1}(u) & \ldots & t_{N N}(u)
\end{array}\right|
$$

for $1 \leqslant i<j \leqslant N$. The Gaussian generators $h_{i}(u), e_{i j}(u)$ and $f_{j i}(u)$ are defined by using the decomposition (2.6) dual to (A.1), and are given by the respective dual quasideterminant formulas; see [19, Section 4]. On the other hand, the mapping

$$
\text { ऽ: } t_{i j}(u) \mapsto t_{i^{\prime} j^{\prime}}(u), \quad 1 \leqslant i, j \leqslant N,
$$

defines an involutive automorphism of the algebra $Y(\mathfrak{g})$. Since quasideterminants are unchanged under permutations of rows or columns, we find that the images of the Gaussian generators are given by

$$
\varsigma: h_{i}(u) \mapsto \bar{h}_{i^{\prime}}(u), \quad e_{i j}(u) \mapsto \bar{f}_{i^{\prime} j^{\prime}}(u), \quad f_{j i}(u) \mapsto \bar{e}_{j^{\prime} i^{\prime}}(u)
$$


Furthermore, the unitary condition (2.5) implies symmetry relations for the Gaussian generators which were described in $\left[19\right.$, Section 5.3]. ${ }^{5}$ They are given in $(2.7)$, and for $i=1, \ldots, n-1$ we have

$$
e_{(i+1)^{\prime} i^{\prime}}(u)=-e_{i i+1}(u+\kappa-i) \quad \text { and } \quad f_{i^{\prime}(i+1)^{\prime}}(u)=-f_{i+1 i}(u+\kappa-i),
$$

with some additional type-specific relations. These relations allow us to express the images of the generators of $\mathrm{Y}^{D}(\mathfrak{g})$ under the composition of the isomorphism $\mathrm{Y}^{D}(\mathfrak{g}) \rightarrow \mathrm{Y}(\mathfrak{g})$ provided by [19, Main Theorem] with the automorphism (A.5), in terms of the Gaussian generators (A.2)-(A.4). The formulas given in the statement of the theorem are obtained by taking further compositions with the shift automorphism $T(u) \mapsto T(u+\kappa-1)$ and with the automorphism which multiplies all generators $\xi_{i r}^{ \pm}$by -1 , while leaving all $\kappa_{i r}$ unchanged.

\section{Acknowledgements}

We acknowledge the support of the Australian Research Council, grant DP180101825.

\section{References}

[1] Arnaudon D., Molev A., Ragoucy E., On the $R$-matrix realization of Yangians and their representations, Ann. Henri Poincaré 7 (2006), 1269-1325, arXiv:math.QA/0511481.

[2] Bazhanov V.V., Trigonometric solutions of triangle equations and classical Lie algebras, Phys. Lett. B 159 (1985), 321-324.

[3] Bazhanov V.V., Integrable quantum systems and classical Lie algebras, Comm. Math. Phys. 113 (1987), 471-503.

[4] Beck J., Braid group action and quantum affine algebras, Comm. Math. Phys. 165 (1994), 555-568, arXiv:hep-th/9404165.

[5] Brundan J., Kleshchev A., Parabolic presentations of the Yangian $Y\left(\mathfrak{g l}_{n}\right)$, Comm. Math. Phys. 254 (2005), 191-220, arXiv:math.QA/0407011.

[6] Chari V., Pressley A., A guide to quantum groups, Cambridge University Press, Cambridge, 1994.

[7] Chari V., Pressley A., Quantum affine algebras and their representations, in Representations of Groups (Banff, AB, 1994), CMS Conf. Proc., Vol. 16, Amer. Math. Soc., Providence, RI, 1995, 59-78, arXiv:hepth/9411145.

[8] Ding J.T., Frenkel I.B., Isomorphism of two realizations of quantum affine algebra $U_{q}(\mathfrak{g l}(n))$, Comm. Math. Phys. 156 (1993), 277-300.

[9] Drinfeld V.G., Hopf algebras and the quantum Yang-Baxter equation, Soviet Math. Dokl. 32 (1985), 254258.

[10] Drinfeld V.G., Quantum groups, in Proceedings of the International Congress of Mathematicians, Vols. 1, 2 (Berkeley, Calif., 1986), Amer. Math. Soc., Providence, RI, 1987, 798-820.

[11] Drinfeld V.G., A new realization of Yangians and of quantum affine algebras, Soviet Math. Dokl. 36 (1988), $212-216$.

[12] Frenkel I.B., Reshetikhin N.Yu., Quantum affine algebras and holonomic difference equations, Comm. Math. Phys. 146 (1992), 1-60.

[13] Gel'fand I.M., Retakh V.S., A theory of noncommutative determinants and characteristic functions of graphs, Funct. Anal. Appl. 26 (1992), 231-246.

[14] Gow L., Molev A., Representations of twisted q-Yangians, Selecta Math. (N.S.) 16 (2010), 439-499, arXiv:0909.4905.

[15] Guay N., Regelskis V., Wendlandt C., Equivalences between three presentations of orthogonal and symplectic Yangians, Lett. Math. Phys. 109 (2019), 327-379, arXiv:1706.05176.

\footnotetext{
${ }^{5}$ The formulas in [19, Proposition 5.7] concerning the Lie algebra $\mathfrak{o}_{2 n+1}$ should be corrected as follows: $e_{n+1} n+2(u)=-e_{n}(u-1 / 2)$ and $f_{n+2} n+1(u)=-f_{n}(u-1 / 2)$.
} 
[16] Hernandez D., Representations of quantum affinizations and fusion product, Transform. Groups 10 (2005), 163-200, arXiv:math.QA/0312336.

[17] Jimbo M., A q-difference analogue of $U(\mathfrak{g})$ and the Yang-Baxter equation, Lett. Math. Phys. 10 (1985), 63-69.

[18] Jimbo M., Quantum $R$ matrix for the generalized Toda system, Comm. Math. Phys. 102 (1986), 537-547.

[19] Jing N., Liu M., Molev A., Isomorphism between the $R$-matrix and Drinfeld presentations of Yangian in types B,C and D, Comm. Math. Phys. 361 (2018), 827-872, arXiv:1705.08155.

[20] Jing N., Liu M., Molev A., Isomorphism between the $R$-matrix and Drinfeld presentations of quantum affine algebra: type $C$, J. Math. Phys. 61 (2020), 031701, 41 pages, arXiv:1903.00204.

[21] Jing N., Liu M., Molev A., Isomorphism between the $R$-matrix and Drinfeld presentations of quantum affine algebra: types $B$ and D, SIGMA 16 (2020), 043, 49 pages, arXiv:1911.03496.

[22] Khoroshkin S., Pakuliak S., Tarasov V., Off-shell Bethe vectors and Drinfeld currents, J. Geom. Phys. 57 (2007), 1713-1732, arXiv:math.QA/0610517.

[23] Kulish P.P., Sklyanin E.K., Quantum spectral transform method. Recent developments, in Integrable Quantum Field Theories (Tvärminne, 1981), Lecture Notes in Phys., Vol. 151, Springer, Berlin - New York, 1982, 61-119.

[24] Molev A., Yangians and classical Lie algebras, Mathematical Surveys and Monographs, Vol. 143, Amer. Math. Soc., Providence, RI, 2007.

[25] Reshetikhin N.Yu., Semenov-Tian-Shansky M.A., Central extensions of quantum current groups, Lett. Math. Phys. 19 (1990), 133-142.

[26] Reshetikhin N.Yu., Takhtadzhyan L.A., Faddeev L.D., Quantization of Lie groups and Lie algebras, Leningrad Math. J. 1 (1990), 193-225.

[27] Tarasov V.O., Structure of quantum $L$-operators for the $R$-matrix of the $X X Z$-model, Theoret. and Math. Phys. 61 (1984), 1065-1072.

[28] Tarasov V.O., Irreducible monodromy matrices for the $R$-matrix of the $X X Z$-model and local lattice quantum Hamiltonians, Theoret. and Math. Phys. 63 (1985), 440-454.

[29] Wendlandt C., The $R$-matrix presentation for the Yangian of a simple Lie algebra, Comm. Math. Phys. 363 (2018), 289-332, arXiv:1709.08162.

[30] Zamolodchikov A.B., Zamolodchikov A.B., Factorized $S$-matrices in two dimensions as the exact solutions of certain relativistic quantum field theory models, Ann. Physics 120 (1979), 253-291. 